\title{
First two operational years of the electron-beam ion trap charge breeder at the National Superconducting Cyclotron Laboratory
}

\author{
A. Lapierre, G. Bollen, D. Crisp, S. W. Krause, L. E. Linhardt, K. Lund, S. Nash, R. Rencsok, \\ R. Ringle, S. Schwarz, M. Steiner, C. Sumithrarachchi, T. Summers, A. C. C. Villari, \\ S. J. Williams, ${ }^{*}$ and Q. Zhao \\ National Superconducting Cyclotron Laboratory, Michigan State University, \\ 640 South Shaw Lane, East Lansing 48824, USA
}

(Received 31 December 2017; published 31 May 2018)

\begin{abstract}
The electron-beam ion trap (EBIT) charge breeder of the ReA post-accelerator, located at the National Superconducting Cyclotron Laboratory (Michigan State University), started on-line operation in September 2015. Since then, the EBIT has delivered many pilot beams of stable isotopes and several rare-isotope beams. An operating aspect of the ReA EBIT is the breeding of high charge states to reach high reaccelerated beam energies. Efficiencies in single charge states of more than $20 \%$ were measured with ${ }^{39} \mathrm{~K}^{15+},{ }^{85} \mathrm{Rb}^{27+},{ }^{47} \mathrm{~K}^{17+}$, and ${ }^{34} \mathrm{Ar}^{15+}$. Producing high charge states demands long breeding times. This reduces the ejection frequency and, hence, increases the number of ions ejected per pulse. Another operating aspect is the ability to spread the distribution in time of the ejected ion pulses to lower the instantaneous rate delivered to experiments. Pulse widths were stretched from a natural $25 \mu \mathrm{s}$ up to $\sim 70 \mathrm{~ms}$. This publication reviews the progress of the ReA EBIT system over the years and presents the results of charge-breeding efficiency measurements and pulse-stretching tests obtained with stable- and rare-isotope beams. Studies performed with high sensitivity to identify and quantify stable-isotope contaminants from the EBIT are also presented, along with a novel method for purifying beams.
\end{abstract}

DOI: 10.1103/PhysRevAccelBeams.21.053401

\section{INTRODUCTION}

The ReA post-accelerator of rare isotopes at the National Superconducting Cyclotron Laboratory (NSCL), located at Michigan State University (MSU), currently accelerates beams up to $\sim 6 \mathrm{MeV} / \mathrm{u}$ for light ions and $\sim 3 \mathrm{MeV} / \mathrm{u}$ for heavy ions (see [1-5] and Fig. 1). ReA ended the commissioning phase and started on-line operation in the fall of 2015. It is the unique post-accelerator reaccelerating rare isotopes thermalized in a gas cell after production by fast projectile fragmentation [6]. One of its essential components is an electron-beam ion trap (EBIT). Compared to other techniques such as the use of stripper targets or electron cyclotron resonance ion sources, electron-beam ion sources (EBISes) and EBITs have been increasingly recognized over the past decades as the best alternatives to charge breeding owing to their high efficiencies in single charge states, small (transverse and longitudinal) emittances, and high beam purity. EBIS/T

\footnotetext{
*Present address: Diamond Light Source Ltd, Harwell Science and Innovation Campus, Didcot, Oxfordshire OX11 0DE, UK.

Published by the American Physical Society under the terms of the Creative Commons Attribution 4.0 International license. Further distribution of this work must maintain attribution to the author(s) and the published article's title, journal citation, and DOI.
}

systems are currently in operation at other rare-isotope facilities such as CARIBU (ANL) [7], TITAN (TRIUMF) [8], and ISOLDE (CERN) [9,10]. More, such as CANREB (TRIUMF) [11], RAON/RISP [12], MATS (GSI/FAIR) [13], EURISOL [14], and HIE-ISOLDE (CERN) [15] are under construction or being considered. For recent reviews of EBIS/T charge breeders, see $[9,10]$.

Commissioning of the ReA EBIT, in its final configuration with the superconducting magnet, started with injection of continuous beams in late 2010. First reacceleration of stable-isotope beams produced from injected gas was realized in 2012 [3]. Reacceleration of stable-isotope beams injected from the thermalized-beam area followed in early 2013 [3,16]. The ReA EBIT was brought on-line for the first time as part of commissioning in late 2013 [17]. It began on-line operation in September 2015 with the addition of an ion beam cooler and buncher (BCB) device for injection of pulsed beams. During the past two years, the EBIT delivered various rare-isotope beams (e.g., ${ }^{46} \mathrm{Ar}$, ${ }^{46} \mathrm{~K},{ }^{34} \mathrm{Ar},{ }^{47} \mathrm{~K},{ }^{37} \mathrm{~K}$ ) for the experimental nuclear-physics program, interlaced with pilot beams of stable isotopes for injection and ejection studies, beam transport optimization, and calibration of detection systems. This publication reviews the progress of the ReA EBIT over the years. It presents the results of efficiency measurements, recent developments on stretching the pulse width of ejected 


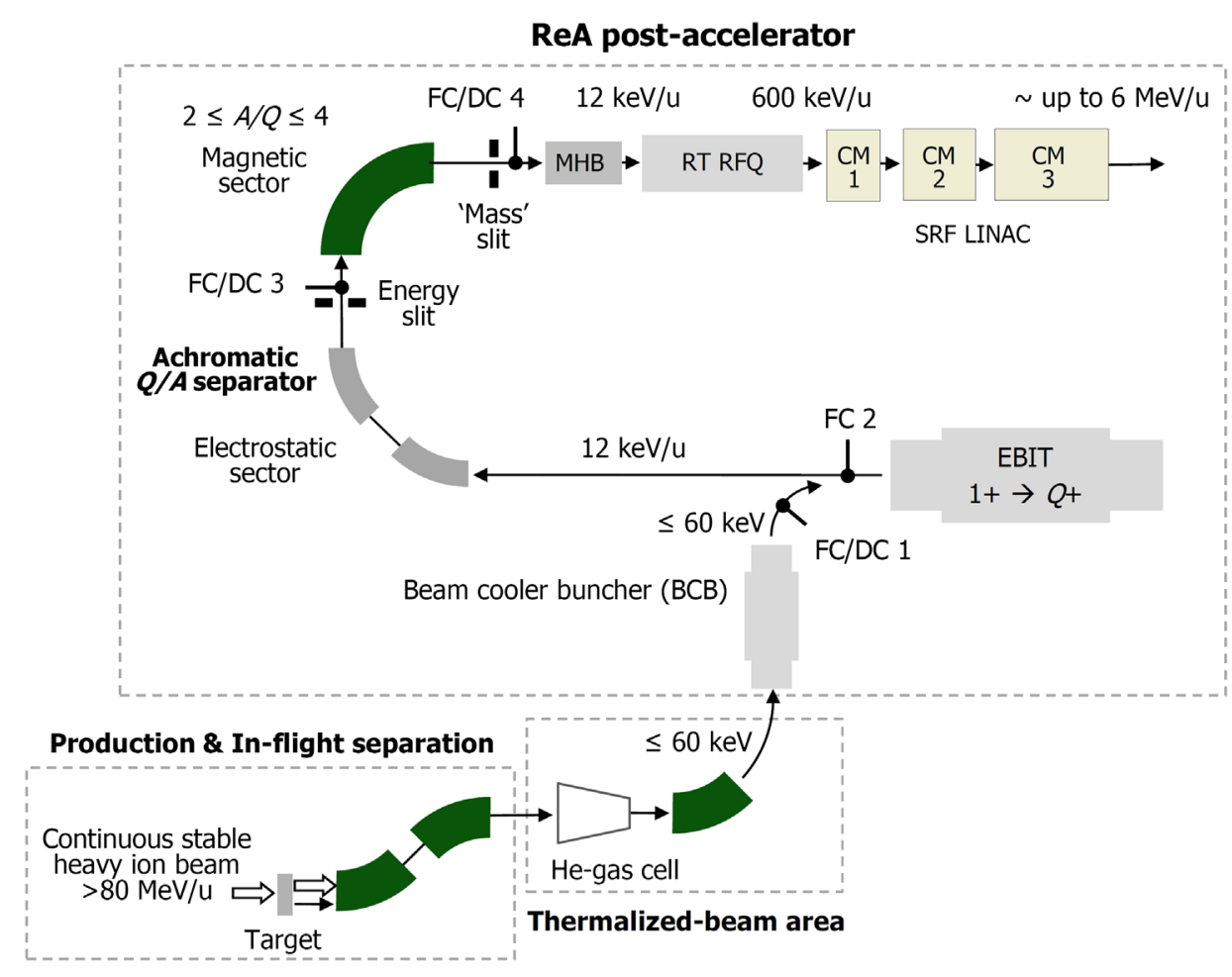

FIG. 1. Schematic layout of the ReA facility, including the beam cooler-buncher (BCB), the EBIT, and the thermalized-beam area, in which the rare-isotope beams produced by projectile fragmentation are thermalized in an He-gas cell. The ion beams are injected into the $\mathrm{BCB}$ as continuous beams where they are bunched and ejected as pulsed beams for injection into the EBIT. After charge breeding, the pulsed highly charged ion beams are transported to an achromatic $Q / A$ separator for subsequent injection into a multiharmonic buncher (MHB), a room-temperature (RT) radio-frequency quadrupole (RFQ) accelerator, and a superconducting rf linear accelerator (SRFLINAC), composed of cryo-modules (CM). Stable-isotope beams of alkali-earth elements are produced from surface-ionization sources installed in the thermalized-beam area as well as on the BCB. FC and DC indicate the location of Faraday cups and $\beta$-decay counters for beam transport optimization and efficiency measurements.

beams, high-sensitivity measurements of ion-beam contaminants, and a new technique to reduce the level of contamination in beams delivered to experiments.

\section{DESIGN AND OPERATION}

\section{A. The EBIT and $Q / A$ separator}

The original design parameters of the ReA EBIT are presented in [3,18]. A schematic layout of the ReA facility, including the EBIT, $\mathrm{BCB}$, and charge-over-mass $(Q / A)$ separator, is shown in Fig. 1. Here, $A$ is the atomic mass in mass unit $\mathrm{u}$, and $Q$ is the ion charge in unit of elementary charge e. A recent photograph of the EBIT can be seen in Fig. 2.

The ReA EBIT was initially designed for continuous ionbeam injection, particularly for beams of high intensity. This is because in a buncher as the number of ions being confined by the trapping potentials increases, the net electric charge of the collection of ions (space charge) also increases. The force that this space charge exerts on the individual ions counteracts the confining forces and, as a consequence, increases the physical size of the ion cloud. The ion-cloud size increases with the intensity of the beam injected into the buncher. This increases the transverse and longitudinal emittances of its ejected pulses, which for high intensities can increase beyond the EBIT acceptance parameters, reducing the injection efficiency into the EBIT. As the intensity further increases, the diameter of the ion

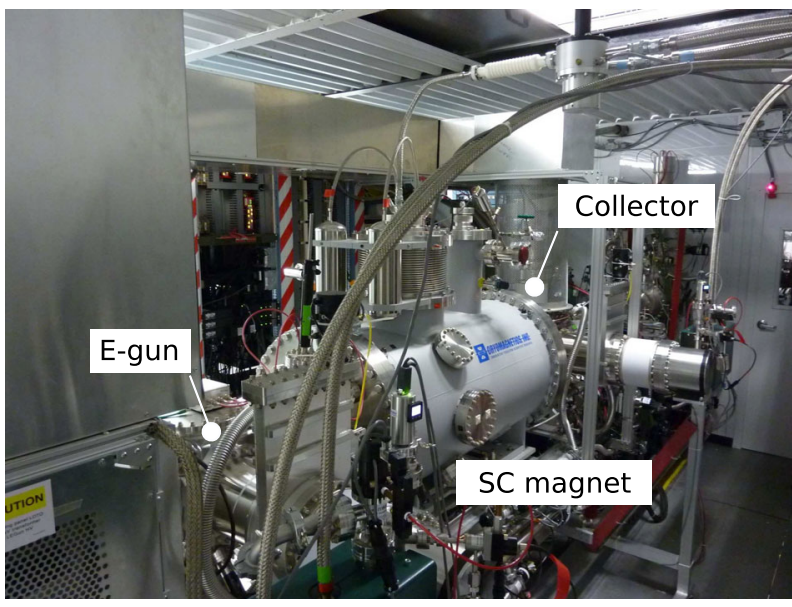

FIG. 2. Photograph of the ReA EBIT. SC stands for superconducting. 
cloud can become larger than the inner diameter of the buncher's trapping electrodes, generating ion losses. Continuous ion-beam injection was initially preferred to overcome such limitations for high-intensity beams of an upstream buncher device. To maximize the beam acceptance, charge capacity, and capture efficiency, the EBIT was built with a high-current electron gun to provide more than one Ampere, a two-segment magnet allowing for multiplefield configurations, and a long trapping region of more than $0.5 \mathrm{~m}$. The magnet is composed of a pair of coils in the (quasi-) Helmholtz configuration and a long solenoid.

With an early electron-gun design, an electron-beam current of nearly 1 A was transported with low electronbeam loss through a pair of room-temperature Helmholtztype coils, used for commissioning, providing a low on-axis field in its center of $\sim 0.25 \mathrm{~T}$ [18]. After installation of the superconducting magnet, however, attempts to transport high currents through a higher field of $4 \mathrm{~T}$ resulted in significant losses caused by reflected electrons returning from the high-field region to the anode. This limited the electron-beam current to less than $\sim 250 \mathrm{~mA}$ within operational parameters deemed safe for the equipment. The electron gun was later redesigned, allowing for transport of up to $1.4 \mathrm{~A}$ through a 4-T field. This latest design is currently employed and is presented in [19].

Once the ions are charge bred, they are ejected and separated in the $Q / A$ separator. The separator allows for charge-state selection of isotopes for subsequent acceleration through the RFQ and superconducting LINAC. The separator is a double-focusing achromatic spectrometer in the Nier-Johnson geometry, composed of 90-deg angle electrostatic and magnetic sectors. Simulations for its design and construction were published in [20], in which, assuming an emittance containing $87 \%$ of the beam ions of $70 \mathrm{~mm} \mathrm{mrad}$ at $12 \mathrm{keV} / \mathrm{u}$, the resolving power of the separator was simulated to be 150 . Using an electron-beam current of $300 \mathrm{~mA}$, the resolving power was recently measured with residual-gas ${ }^{40} \mathrm{Ar}^{17+}$ and ${ }^{14} \mathrm{~N}^{6+}$ ejected with a 10-ms linear voltage ramp function (see Sec. V) and an acceleration voltage of $38 \mathrm{kV}$. The resolving power was measured to be approximately 400 for a beam width containing $90 \%$ of the beam ions of $\sim 2.4 \mathrm{~mm}$. Performing a scaling from the simulations, this would indicate a beam emittance of $\sim 7 \mathrm{~mm}$ mrad. In previous measurements performed with an electron-beam current of $800 \mathrm{~mA}$, the $Q / A$ separator was used to measure the energy spread of ${ }^{16} \mathrm{O}^{7+}$ and ${ }^{39} \mathrm{~K}^{15+}$ beams ejected from the ReA EBIT to be $31 \mathrm{eV} / \mathrm{e} \times Q$ and $25 \mathrm{eV} / \mathrm{e} \times Q$, respectively. This energy spread was inferred from the temperature of the trapped ions by fitting the intensity profile obtained after the energy sector with an energy distribution. The details of the energy-spread measurements were published in [21]. Note that the ratio of the energy spread to the beam energy $(\Delta E / E)$ during the present charge-breeding measurements was well within the energy acceptance of $\Delta E / E \sim 3 \%$ of the separator, and did not affect the measurements. For optimizing transport of stable as well as rare-isotope beams, the separator includes Faraday cups (see Fig. 1) located at the exit of the electrostatic sector (FC/DC 3 ) and the exit of the magnetic sector (FC/DC 4), which are called here energy and mass slits, respectively. Each Faraday cup incorporates a silicon detector for $\beta$-decay counting the activity of beam ions deposited onto an aluminum foil of less than $\sim 25 \mu \mathrm{m}$ in thickness [22]. All Faraday cups are equipped with a front-ring electrode to suppress secondary electrons.

A recent addition to the ReA EBIT is a large acceptance Einzel lens with an inner diameter of $100 \mathrm{~mm}$. The lens is part of the acceleration gap, shown in Fig. 3. The central section of the lens is split into four individual segments with two diagonal cuts, rotated by $90 \mathrm{deg}$, to add 2-dimensional (horizontal and vertical, $x-y$ ) steering capabilities [23]. With sets of horizontal and vertical steerers in the injection/ejection beam line, this "split" lens allows for parallel corrections to the trajectory of injected and ejected beams. This is needed to optimize transport through the collector for injection into the electrode structure of the trap (and magnetic field) and through the $Q / A$, respectively. In the design of the lens, SIMION [24] simulations were performed assuming an ejected beam of a transverse rootmean-square (rms) emittance of $10 \mathrm{~mm}$ mrad with an energy $30 \mathrm{keV} / \mathrm{e} \times Q$. See [25] for comments on the definition of emittance. Using this value for the transverse emittance in the simulations is considered reasonable. Assuming that trapped ions of a temperature of $1 \mathrm{keV}$ are encompassed within an electron beam of an $80 \%$ radius of $200 \mu \mathrm{m}$ in a 4-T field [26], the expected (rms) emittance of a ${ }^{39} \mathrm{~K}^{10+}$ beam ejected with an acceleration voltage of $30 \mathrm{kV}$ is estimated to be $6 \mathrm{~mm}$ mrad [27,28]. This emittance estimate includes the effect of the magnetic field. From the results of our SIMION simulations, the new large acceptance lens is expected to introduce only a small emittance growth of approximately $2.5 \%$ for an ejected (on-axis) beam emittance of $10 \mathrm{~mm}$ mrad with an energy of $30 \times 10=300 \mathrm{keV}$. The advantage of diagonal-split deflectors in reducing emittance growth, compared to parallel deflectors for instance, was discussed in $[23,28]$.

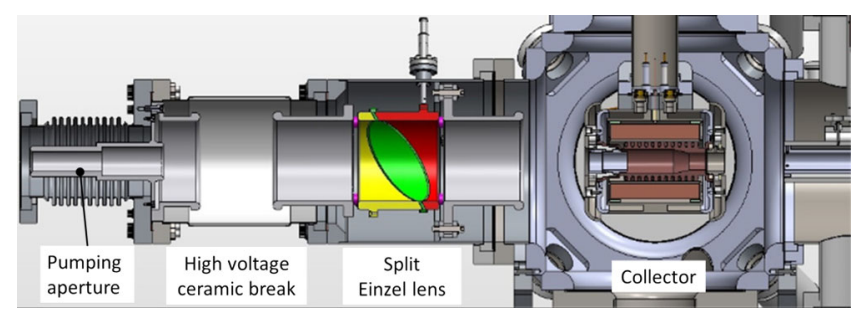

FIG. 3. Rendering cut of a model of the high-acceptance "split" Einzel lens recently installed near the acceleration gap to reduce emittance growth and allow for horizontal and vertical beam steering during injection and ejection. 


\section{B. The beam cooler and buncher}

The capture efficiency of ion beams continuously injected into an EBIS/T is highly dependent on the electron-beam current and current density. This is because they both governs, respectively, the geometrical acceptance of the device and the ionization probability of the injected ions, which have to be ionized to a higher charge state while performing the first round trip in the trap. Acceptance calculations with a simple geometrical model and Monte Carlo simulations were compared with experimental results in $[29,30]$. Moreover, during continuous ion injection, unlike pulsed injection in which all ions within a pulse width enter the trap at approximately the same time, not all ions experience the same breeding time. This broadens the charge-state distribution and, hence, reduces the breeding efficiency in single charge states [17].

A helium gas-filled radio-frequency quadrupole Paul trap used as a BCB was recently incorporated into ReA to add the capability of injecting pulsed beams (ion pulses) into the ReA EBIT to improve the capture and breeding efficiencies with low electron-beam currents. The BCB is divided into upstream and downstream sections, the latter including the ion bunching part of the device. These upstream and downstream sections operate at radio frequencies of 5 and $4 \mathrm{MHz}$, respectively. The ReA $\mathrm{BCB}$ is close in design to the beam cooler buncher of the BECOLA facility [31]. The BCB was commissioned in the summer of 2015, and started on-line operation at the same time as the ReA EBIT. The pulsed beams from the $\mathrm{BCB}$ are produced by accumulating continuous beams from either one of three ion sources: its dedicated test "alkali" or surface-ionization source, a similar one placed in the helium-gas cell (in the thermalized-beam area) for stable isotopes (pilot beams) and the helium-gas cell for the rare isotopes. The BCB was specifically designed for high beam rates. Emittance measurements indicate that up to $\sim 10^{7}$ ions per pulse can be bunched with a transverse (rms) emittance below $\sim 10 \mathrm{~mm}$ mrad at an ejected beam energy of $30 \mathrm{keV}$. Preliminary measurements of the width of pulses ejected from the BCB showed the widths to be within $5 \mu \mathrm{s}$. The efficiency of the BCB for pulsed beams was measured with stable- and rare-isotope beams to be typically better than $50 \%$. This is being investigated. Located directly at the exit of the BCB is a Faraday cup (FD/DC 1 in Fig. 1) including a silicon detector as decay counter. This assembly is of the same design as those in the $Q / A$ separator, mentioned earlier.

\section{Operational parameters}

Operational parameters of the ReA EBIT used for injection, breeding, ejection, and reacceleration of stableand rare-isotope beams delivered to experiments are presented in Table I. The maximum field of the magnet is $6 \mathrm{~T}$. A $4 \mathrm{~T}-4 \mathrm{~T}$ field configuration (Helmholtz coils - solenoid) was chosen for operation as a compromise to facilitate
TABLE I. Typical ReA EBIT operational parameters employed for reacceleration of beams to experiments. "SCS efficiency" stands for the charge-breeding efficiency in single charge states. $A$ and $Q$ are the mass and charge of the reaccelerated ion in units of $u$ and e, respectively. The electron-beam current density was estimated from the electron-beam radius measured by $\mathrm{x}$-ray imaging (see Sec. II C).

\begin{tabular}{ll}
\hline Injection/Ejection voltage & $12 \mathrm{keV} / \mathrm{u} \times A / Q$ \\
Injection pulse width & $\leq 5 \mu \mathrm{s}$ \\
Injection energy & $12 \mathrm{keV} / \mathrm{u} \times A e / Q$ \\
Ejection energy & $12 \mathrm{keV} / \mathrm{u} \times A$ \\
SCS efficiency & $\sim 10-25 \%$ \\
Breeding time & $\leq 400 \mathrm{~ms}$ \\
Ejection time & $\leq 125 \mathrm{~ms}$ \\
Ejection pulse widths & $\sim 20 \mu \mathrm{s}-100 \mathrm{~ms}$ \\
Ejection beam current & $300-600 \mathrm{~mA}$ \\
Ejection beam density & $170-340 \mathrm{~A} / \mathrm{cm}^{2}$ \\
Ejection beam energy & $15.5 \mathrm{keV}$ \\
Field configuration & $4 \mathrm{~T}(\mathrm{coils})-4 \mathrm{~T}$ (sol.) \\
Length of trapping region & $0.64 \mathrm{~m}$ \\
\hline \hline
\end{tabular}

transport of high electron-beam currents to increase the acceptance and for high compression to augment the current density. Operation with high electron-beam currents, however, can be unstable. For the sake of stability during weeks of continuous 24/7 operation, moderate electron-beam currents between 300 and $600 \mathrm{~mA}$ are normally chosen. The ReA EBIT operates with a continuous electron beam. In [26], the radius of an 800-mA electron beam, provided by the latest electron-gun design [19], containing $80 \%$ of the electron current was determined in a 4-T field by $\mathrm{x}$-ray imaging to be $212(19) \mu \mathrm{m}$. From these values, a current density of $454(83) \mathrm{A} / \mathrm{cm}^{2}$ could be deduced. Herrmann theory $[32,33]$ predicts that for our electron gun and with an electron-beam energy of tens of $\mathrm{keV}$, the electron-beam radius only varies within $0.1 \%$ as the electron-beam current is reduced from 800 to $300 \mathrm{~mA}$. As a result, assuming a constant radius, the current densities employed for operation are estimated to be between 170 and $340 \mathrm{~A} / \mathrm{cm}^{2}$ (see Sec. IV).

\section{Injection, trapping, and ejection}

The fixed injection velocity into the ReA RFQ corresponding to $12 \mathrm{keV} / \mathrm{u}$ defines the overall beam transport energy from the thermalized-beam area up to the EBIT and the RFQ.

In a full cycle, a continuous beam of singly charged ions of $12 \mathrm{keV} / \mathrm{u} \times A / Q e$ ( $A$ and $Q$ are the mass and charge, respectively, of the reaccelerated ion) is injected from the helium-gas cell into the $\mathrm{BCB}$, where the ions are decelerated, accumulated, and cooled. At the end of an RFQ ontime period, which corresponds to $t=0$ in the sequence illustrated in Fig. 4, the RFQ pulse delay generator (PDG) triggers the EBIT PDG, which prompts the BCB PDG, causing the opening of the $\mathrm{BCB}$ trap for the release of an 


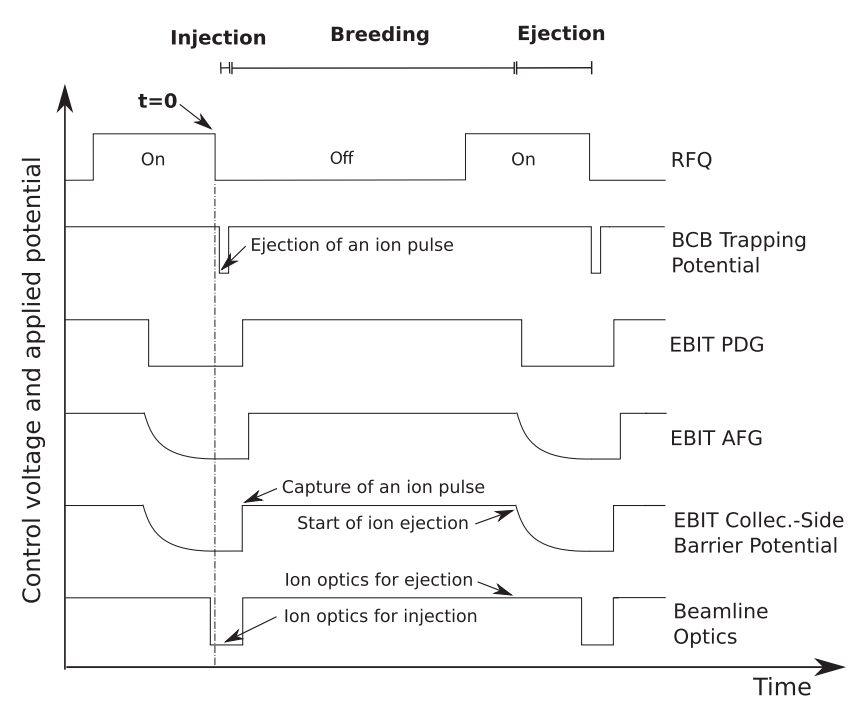

FIG. 4. Time sequence for ejection of ion pulses from the BCB, capture of those pulses into the ReA EBIT for charge breeding, and ion release with a voltage ramp for subsequent injection into the ReA RFQ for acceleration. The falling edge of the RFQ ontime period $(t=0)$ triggers the EBIT pulse delay generator (PDG), which then prompts ion ejection from the $\mathrm{BCB}$, and the following actions in the sequence.

ion pulse. An ion pulse is then ejected, accelerated, and transported to the EBIT at $12 \mathrm{keV} / \mathrm{u} \times A / Q e$ for injection.

For injection, trapping, and ejection, the EBIT's highvoltage platform is biased at a constant potential, which is $3 \mathrm{kV}$ lower than the beam transport energy from the BCB. During injection, all electrostatic elements upstream the EBIT switch to injection optics settings and the EBIT's trapping barrier potential on the collector side is kept lower than or near the potential in the trap center for the incoming pulse to enter the trapping region. The injection, ejection, and trapping potential configurations of the ReA EBIT are displayed in Fig. 5. Upon injection, the ion pulse is decelerated, focused as well as steered by the split Einzel lens in the acceleration gap, and injected into the trapping region, through the collector.

Depending on the electron-beam current, the trap center is typically biased at a potential between 3.125 and $3.250 \mathrm{kV}$ above the voltage applied to the EBIT's highvoltage platform (see Fig. 5). The space-charge potential in the center of the electron beam, calculated from its central axis to the inner surface of the trapping electrodes, is between $-278 \mathrm{~V}(300 \mathrm{~mA})$ and $-555 \mathrm{~V}(600 \mathrm{~mA})$. From these potentials, the initial kinetic energy of the injected ions in the trap can be calculated to be less than $\sim 400 \mathrm{eV}$. As the injected pulse reaches the trapping potential barrier on the electron-gun side, the EBIT PDG causes a fast highvoltage amplifier to raise the potential applied to the collector-side barrier and capture the traveling pulse. It also switches elements in the injection beamline to ejection optics settings in preparation for ejection to the $Q / A$ separator.

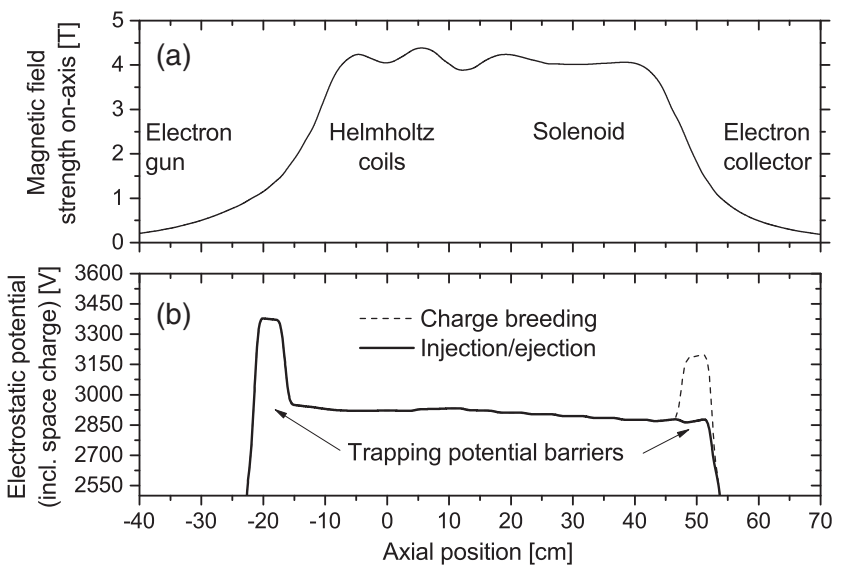

FIG. 5. (a) Magnetic field distribution measured on-axis of the ReA EBIT's magnet composed of a pair of coils in the (quasi-) Helmholtz configuration and a long solenoid, and (b) injection, trapping, and ejection electrostatic potentials, including the space-charge potential on axis of a $300-\mathrm{mA}$ electron beam. The electrostatic potential is non-uniform in the center of the trap due to inhomogeneities in the magnetic field which affect the electron-beam space-charge potential.

The potential applied to the collector-side barrier is normally between 3.3 and $3.5 \mathrm{kV}$, depending on the electron current. The EBIT's trapping potential, defined as the difference between the potential in the center of the trap and that of the collector-side barrier, is normally set to $\sim 250 \mathrm{~V}$. However, it can be adjusted during a beam time to optimize production of the charge state needed for reacceleration. Approximately $3 \mathrm{~ms}$ prior to the end of the charge breeding process, the RFQ PDG turns the RFQ on. After breeding, the EBIT PDG triggers an arbitrary function generator (AFG). Through the high-voltage amplifier, the AFG then lowers the voltage applied to the collector-side barrier electrode with the use of a special waveform for a slow release of ions. Following ejection, the EBIT PDG keeps the trap open in preparation for receiving the next pulse being bunched in the $\mathrm{BCB}$. The ion pulse ejected from the EBIT is then accelerated to an energy of $12 \mathrm{keV} / \mathrm{u} \times A$, transported to the $Q / A$ separator for charge-state selection, and subsequently injected into the RFQ.

\section{EFFICIENCY STUDIES}

\section{A. Stable isotopes}

\section{Measurements and method}

The charge-breeding efficiency of the stable isotopes ${ }^{39} \mathrm{~K},{ }^{85} \mathrm{Rb}$, and ${ }^{133} \mathrm{Cs}$ was measured with electron-beam currents of 302, 333, and $573 \mathrm{~mA}$, respectively, and an electron-beam energy of $15.5 \mathrm{keV}$ (excluding the contribution of the electron-beam space-charge potential).

Pulsed beams were injected into the EBIT from the BCB that was continuously fed with stable-isotope beams from 
one of the two test ion sources. The ion source installed on the $\mathrm{BCB}$ provided a potassium beam without mass separation, the natural isotopes ${ }^{39} \mathrm{~K}$ and ${ }^{41} \mathrm{~K}$. In the ${ }^{39} \mathrm{~K}$ efficiency calculations, the injected ${ }^{39} \mathrm{~K}$ current was determined by correcting the total injected current for the ${ }^{41} \mathrm{~K}$ natural abundance of $6.7 \%$. The ${ }^{85} \mathrm{Rb}$ beam was produced from the test ion source in the thermalized-beam area and selected with a magnet separator. Cs beam was produced from the test ion source on the BCB containing only the stable isotope ${ }^{133} \mathrm{Cs}$. Some new surface-ionization sources were found to contain alkali contaminants, which would vanish after using the source for several weeks. For instance, $\mathrm{K}$ ion emitters were found to produce $\mathrm{Na}$ ions as well. This would have the effect of increasing the apparent $\mathrm{K}$ and $\mathrm{Cs}$ injected beam currents and, hence, reducing the measured efficiencies. However, no trace of $\mathrm{Na}, \mathrm{K}$, or $\mathrm{Rb}$ contaminants were found in the $\mathrm{K}$ and $\mathrm{Cs}$ $Q / A$ spectra at the epA level.

The emittance of the beam from the BCB grows with the number of ejected ions per pulse. For the purpose of the measurements, a rate of injection into the ReA EBIT of approximately $7 \mathrm{~Hz}$ was selected to limit the number of ions per pulse ejected from the BCB to less than $4 \times 10^{7}$ and, hence, restrict the beam emittance. This translated into breeding and ejection times of 110 and approximately $20 \mathrm{~ms}$. The ammeters utilized with the Faraday cups showed typical noise levels of less than $\pm 2 \mathrm{epA}$, considered here as a $\sim 2-\sigma$ error. Ion beam currents between 10 and 40 epA were injected into the EBIT as a compromise to keep the BCB beam emittance low, but limit the uncertainty of the current measurements by keeping the beam current sufficiently high above the noise levels. Linear $\left({ }^{39} \mathrm{~K}\right.$ and $\left.{ }^{133} \mathrm{Cs}\right)$ and logarithmic $\left({ }^{85} \mathrm{Rb}\right)$ voltage ramp functions were employed for ion ejection from the EBIT (see Sec. V).

For each measurement, the beam currents injected into the EBIT were measured with FC 2 (Fig. 1) and maximized with upstream (horizontal and vertical) correction steerers. The inner diameter of FC 2 is $16.5 \mathrm{~mm}$, while the full beam diameter (containing more than $90 \%$ of the ions) was observed with an MCP (micro-channel plate) viewer to be less than $9 \mathrm{~mm}$. For each efficiency measurement conducted with a $Q / A$ scan, the injected beam current was measured before and after the scan, and the current used in the efficiency calculation was obtained from the average. The variations in current during the scans were normally less than a few epA. Before the efficiency measurements, beam transmission through the EBIT and $Q / A$ separator was optimized by maximizing the beam current in FC/DC 4. The inner diameter of FC/DC 4 is $30 \mathrm{~mm}$, fully capturing the beam with its diameter of less than $6 \mathrm{~mm}$.

All $Q / A$ scans, performed by varying the field strength of the $Q / A$ separator's magnet, were conducted with large openings, between 4 and $6 \mathrm{~mm}$, of the mass slit located at the image point. This was needed for the measured beam current of each charge state to represent its total current.
The opening of the energy slit was kept large as well, between 10 and $15 \mathrm{~mm}$, to prevent ion losses.

\section{Results and discussions}

Figures 6-8 show the $Q / A$ spectra and the corresponding efficiencies in single charge states (SCS) of the injected stable beams being studied. Table II presents the operational parameters used during the measurements. It is important to stress that these efficiency values do not include the BCB efficiency; they represent the EBIT efficiencies, only. The SCS effciencies were calculated from the ejected beam currents $\left(I_{e j}\right)$ measured at the mass slit (FC/DC 4) and the injected ion currents $\left(I_{\text {inj }}\right)$ measured at the EBIT entrance (FC 2) as:

$$
\epsilon_{\mathrm{eff}}=\frac{I_{e j}}{Q} \frac{e}{I_{\mathrm{in}}}
$$

Each figure also displays the efficiency for all charge states (ACS), which corresponds to the efficiency for capture and breeding the injected beam. Measuring ACS efficiencies at the energy slit (FC/DC 3), before the magnetic sector, is not straightforward with stable-isotope beams. The beam current at this location is the total current of all ions ejected from the trap that includes residual-gas ions (e.g., C, N, O, Ar, etc.). The ACS efficiencies were
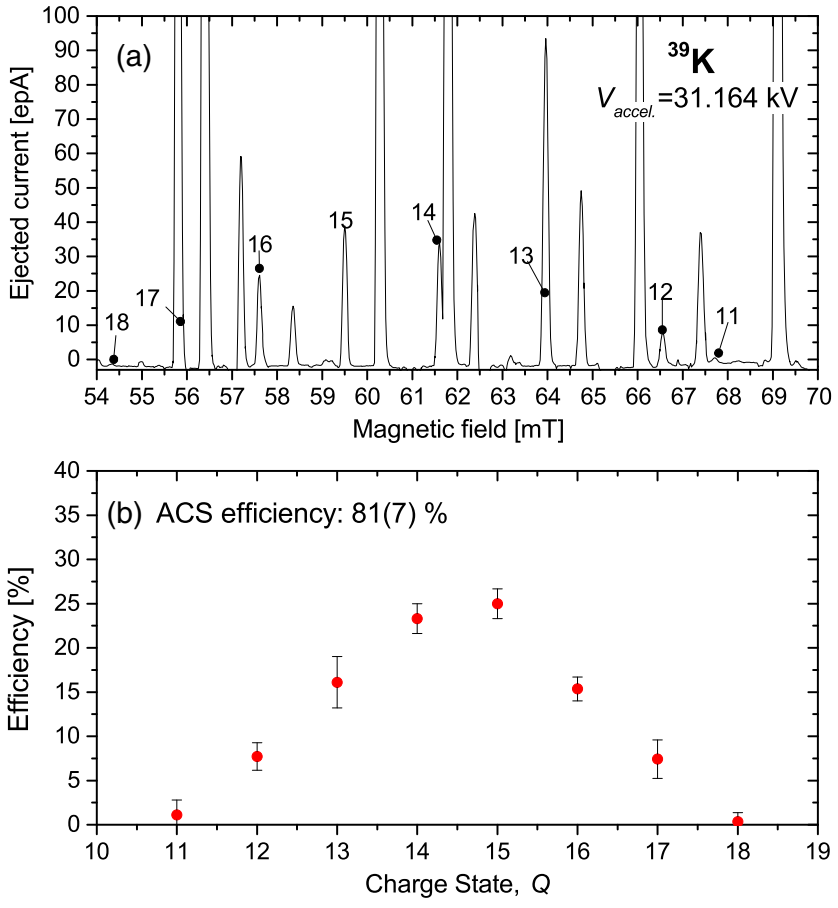

FIG. 6. (a) $Q / A$ scan of ${ }^{39} \mathrm{~K}$ charge states and (b) corresponding efficiency in single charge states. Also included is the all-chargestate (ACS) efficiency calculated by summing up the singlecharge-state (SCS) efficiencies over all the charge states observed within the scanned $Q / A$ region. 

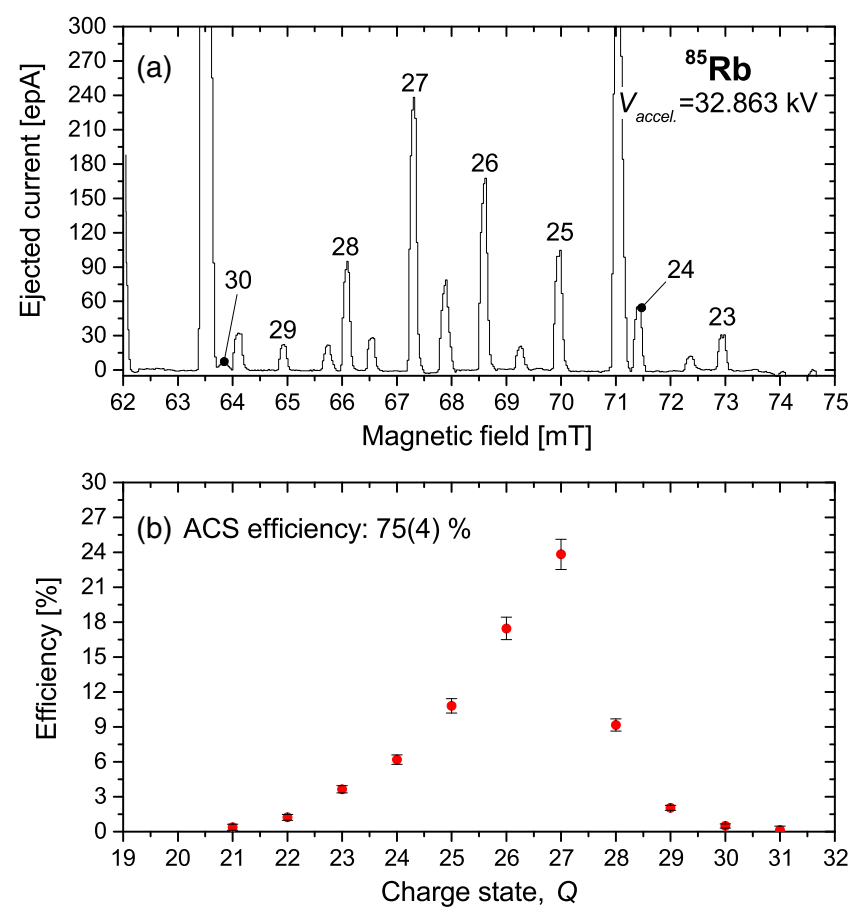

FIG. 7. Same as Fig. 6. but for ${ }^{85} \mathrm{Rb}$.

calculated by summing the SCS efficiencies shown in each figure. All charge states outside the range of the $Q / A$ scans were considered negligible. The uncertainty of the SCS efficiencies results primarily from the \pm 2 -pA error associated to the noise of the Faraday cups and ammeters. The uncertainty of the ACS efficiencies was obtained from
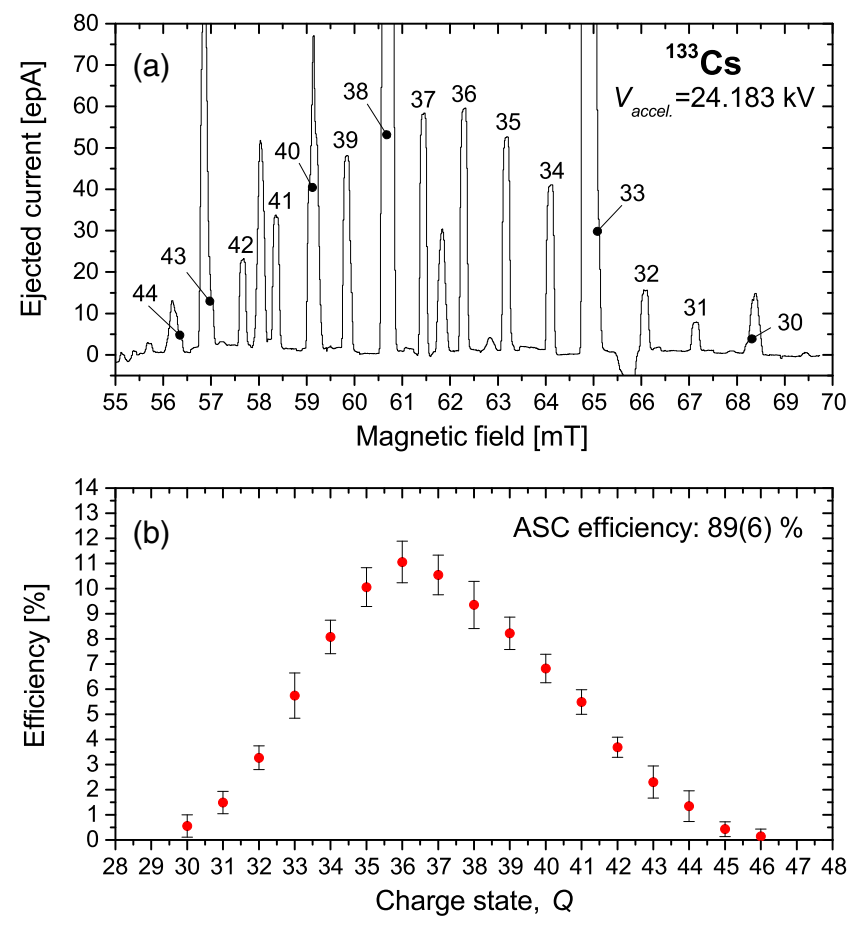

FIG. 8. Same as Fig. 6, but for ${ }^{133}$ Cs.
TABLE II. Maximum single charge state (SCS) and all-chargestate (ACS) efficiencies of stable-isotope beams. The ACS efficiencies were measured at the mass slit (MS) after the separator magnet by integrating the SCS efficiencies obtained in a $Q / A$ scan.

\begin{tabular}{|c|c|c|c|c|c|c|}
\hline $\begin{array}{l}\text { Element } \\
\text { Isotope } \\
\text { (Maximum } \\
\text { CS) }\end{array}$ & $\begin{array}{c}\text { Electron } \\
\text { current } \\
{[\mathrm{mA}]}\end{array}$ & $\begin{array}{c}\text { Injection } \\
\text { current } \\
\text { [epA] }\end{array}$ & $\begin{array}{l}\text { Injection } \\
\text { freq. } \\
\text { [Hz] }\end{array}$ & $\begin{array}{l}\text { Breeding+ } \\
\text { ejection } \\
\text { time }[\mathrm{ms}]\end{array}$ & $\begin{array}{c}\text { Maximum } \\
\text { SCS } \\
\text { efficiency } \\
{[\%]}\end{array}$ & $\begin{array}{l}\text { ACS } \\
\text { efficiency } \\
\text { [\%] (MS) }\end{array}$ \\
\hline${ }^{39} \mathrm{~K}(15+)$ & $302(10)$ & $13(1)$ & 6.98 & $110+23$ & $25(2)$ & $81(7)$ \\
\hline${ }^{85} \mathrm{Rb}(27+)$ & $333(10)$ & $37(2)$ & 7.12 & $110+20$ & $24(1)$ & $75(4)$ \\
\hline${ }^{133} \mathrm{Cs}(36+)$ & $573(10)$ & $15(1)$ & 7.00 & $110+23$ & 11(1) & $89(6)$ \\
\hline
\end{tabular}

those of the SCS efficiencies added in quadrature. Certain ${ }^{39} \mathrm{~K},{ }^{85} \mathrm{Rb}$, and ${ }^{133} \mathrm{Cs}$ charge states were fully blended with charge states of residual-gas ions. The current of these charge states was estimated by calculating the average current of the two adjacent charge states and the error was calculated as twice the error estimated for each adjacent charge state, i.e., 4 pA.

For the operational parameters mentioned earlier, maximum SCS efficiencies of 25(2)\%, 24(1)\%, and 11(1)\% were obtained with ${ }^{39} \mathrm{~K}^{15+},{ }^{85} \mathrm{Rb}^{27+}$, and ${ }^{133} \mathrm{Cs}^{36+}$ with ACS efficiencies of $81(7) \%, 75(4) \%$, and $89(6) \%$, respectively. Simulations with the CBSIM code [34] were conducted with an arbitrary electron-beam current density and a radial potential sufficiently high to avoid ion losses. In a charge-state distribution, a charge-state anchor can be defined as the ratio of the most abundant charge state to the least abundant among the two adjacent charge states. For the experimental charge-state anchor ratios presented in Table IV (Sec. IV), CBSIM predicts for those charge states efficiencies of about 35\%, 30\%, and 22\%. The ACS efficiencies reveal the overall ion losses, which average to $18 \%$, that affected our measurements. Ions were lost in the injection, ejection, and/or breeding process(es). The origin of these losses is currently being investigated. Assuming that each charge state was equally affected, the experimental SCS efficiencies were corrected for the losses by dividing by their respective ACS efficiencies. The measured, corrected SCS efficiencies are $31 \%, 32 \%$, and $12 \%$. The ${ }^{39} \mathrm{~K}$ and ${ }^{85} \mathrm{Rb}$ results are in satisfactory agreement with the simulations. However, our ${ }^{133} \mathrm{Cs}^{36+}$ efficiency is significantly lower than expected by approximately a factor of two.

One can compare our efficiencies with those of the REX EBIS. In [9], Wenander et al. state SCS efficiencies combining the Penning-trap and breeding efficiencies of $19.8 \%, 12.7 \%$, and $10.8 \%$ for ${ }^{39} \mathrm{~K}^{9+},{ }^{87} \mathrm{Rb}^{20+}$, and ${ }^{133} \mathrm{Cs}^{34+}$. The estimated efficiency of the REX Penning trap for these masses is in the range of $60 \%$. Correcting these combined efficiencies, one can estimate REX EBIS-only efficiencies of $33 \%, 21 \%$, and $18 \%$. Our ${ }^{39} \mathrm{~K}$ and ${ }^{85} \mathrm{Rb}$ loss-corrected results are in good agreement with the REX EBIS efficiencies, except, again, for ${ }^{133} \mathrm{Cs}$. The CARIBU EBIS recently obtained an efficiency of $20 \%$ with ${ }^{133} \mathrm{Cs}^{28+}$ [7], which is in close agreement with the REX EBIS result. 

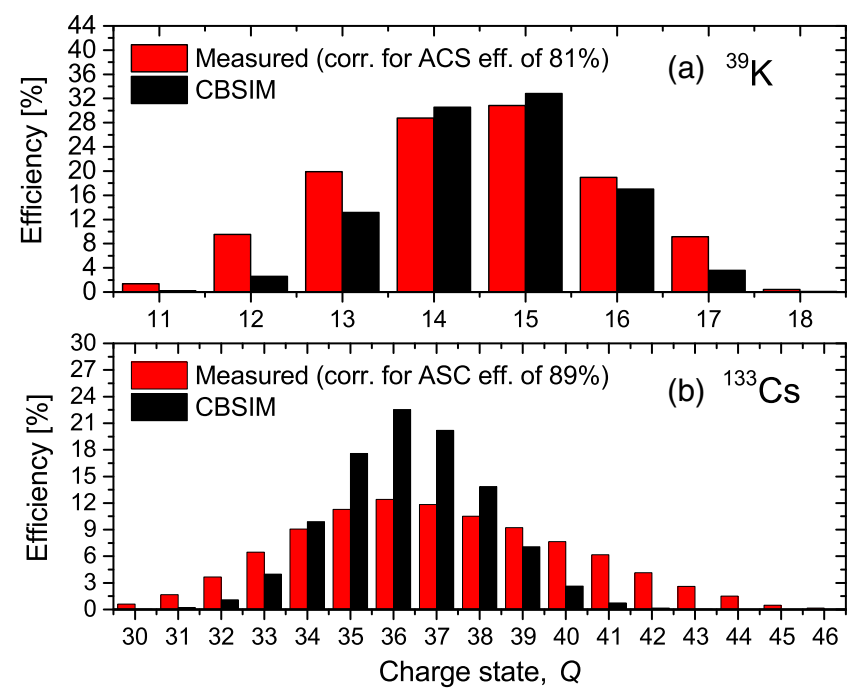

FIG. 9. Measured charge-state distributions compared with CBSIM simulations: (a) ${ }^{39} \mathrm{~K}$ and (b) ${ }^{133} \mathrm{Cs}$. Unlike the ${ }^{133} \mathrm{Cs}$ distribution, the ${ }^{39} \mathrm{~K}$ distribution is slightly broader, but in satisfactory agreement with the simulated distribution (see text for explanation).

The deviation of our ${ }^{133} \mathrm{Cs}$ result appears to be consistent with the broad ${ }^{133} \mathrm{Cs}$ charge-state distribution that we observe. This broad distribution has the effect of reducing the peak efficiency for the $36+$ charge state. This can be seen in Fig. 9, which compares our ${ }^{133} \mathrm{Cs}$ charge-state distribution with a CBSIM-simulated one, and in which the measured distribution was normalized to the ACS efficiency of $89 \%$.

The electrostatic potential applied in the trapping region is normally kept uniform. However, an imperfect voltage calibration of power supplies can create local traps within the entire trapping region. Moreover, as shown in Fig. 5, the magnetic field in the trapping region has inhomogeneities of up to approximately $\pm 3 \%$ of the average central field of $4 \mathrm{~T}$. This modulates the electron-beam space-charge potential and can create shallow local traps. Furthermore, as also shown in Fig. 5, the magnetic field strength at the potential barriers is four times as low as that of the central field. This makes the electron-beam space-charge potential higher in the center of the trap than at the barriers. As a consequence, applying the same electrostatic potential to all electrodes can create a shallow trapping potential that may hinder ejection of all ions if the barrier potential on the collector side is insufficiently lowered. Therefore, one possible explanation for the observed broad ${ }^{133} \mathrm{Cs}$ distribution is that a large portion of the injected ions may have remained confined for a few injection-ejection cycles before being ejected, experiencing longer breeding times as a result.

Note that the charge state having the maximum intensity (SCS efficiency) in a distribution depends on the product of the electron-beam current density $\left(J_{e}\right)$ and the breeding time $\left(\tau_{b}\right)$ as $J_{e} \tau_{b}$. The electron-beam radius being constant for our electron-gun design, changes in the electron current vary the current density proportionally, and affect a chargestate distribution the same way as varying the breeding time. Detailed studies of the dependence of the ACS and SCS efficiencies with the injected charge (i.e., injected current) were not carried out. However, preliminary breeding time measurements with residual gas were conducted to determine the time constant for neutralization of the electron beam. These measurements have shown that after approximately $200 \mathrm{~ms}$, the electron beam reaches a neutralization factor of $50 \%$, remaining rather constant for breeding times of more than $1 \mathrm{sec}$. For the efficiency measurements with long breeding times presented here, the ACS efficiencies may have been reduced due to significant electron-beam neutralization that would have decreased the effective axial and radial trapping potentials experienced by the trapped ions. This is being investigated.

\section{B. Rare isotopes}

\section{Measurements and method}

Over the last two years, rare-isotope beams such as ${ }^{46} \mathrm{Ar}^{17+},{ }^{46} \mathrm{~K}^{18+},{ }^{37} \mathrm{~K}^{17+},{ }^{34} \mathrm{Ar}^{15+}$, and ${ }^{47} \mathrm{~K}^{17+}$ were charge bred and reaccelerated for nuclear-physics experiments. The electron-beam currents ranged from 332 to $569 \mathrm{~mA}$. The beams were injected from the helium-gas cell as continuous beams into the $\mathrm{BCB}$ and then injected into the EBIT as pulsed beams at repetition rates between 2 and $5 \mathrm{~Hz}$. These repetition rates were chosen to maximize production of specific charge states suitable for the reaccelerated beam energy and the beam purity required. Ejection times between 68 and $125 \mathrm{~ms}$ were chosen to optimize the time spread of the ion pulses ejected from the EBIT. This was required to match the "dead" time of the detection systems used by the experiments. Rare-isotope rates of up to one million per second were injected. The efficiencies were obtained by measuring the $\beta$-decay rates of the beams with the decay counters located in the injection beam line (FC/DC 1 ) and $Q / A$ separator $(\mathrm{FC} /$ DC 3 and FC/DC 4). The geometrical detection efficiency of all decay counters is estimated to be $30 \%$, but their design and configuration are identical, allowing for the geometrical efficiency to cancel out. The $Q / A$ scans were conducted with widely opened energy and mass slits.

The beam intensities (or rates) measured at FC/DC 1 were not optimized with upstream steerers because it was shown during stable-isotope beam measurements that the beam at this location was fully captured by the Faraday cup. Nevertheless, the beam intensities were maximized in FC/ DC 4 prior to the $Q / A$ scans (as described in Sec. III A).

\section{Results and discussions}

Table III presents the SCS and ACS efficiencies of the rare-isotope beams. This table includes the electron-beam current used during the runs together with the breeding and 
TABLE III. Single charge state (SCS) and all-charge-state (ACS) efficiencies of rare-isotope beams reaccelerated to nuclear-physics experiments. The ACS efficiencies were measured at two locations: at the energy slit (ES) before the $Q / A$ separator's magnet and at the mass slit (MS) after the magnet.

\begin{tabular}{|c|c|c|c|c|c|c|}
\hline $\begin{array}{l}\text { Element } \\
\text { Isotope }\end{array}$ & $\begin{array}{c}\text { Half-life } \\
\text { [ms] }\end{array}$ & $\begin{array}{c}\text { Electron } \\
\text { current } \\
{[\mathrm{mA}]}\end{array}$ & $\begin{array}{c}\text { Breeding+ } \\
\text { Ejection } \\
\text { time }[\mathrm{ms}]\end{array}$ & $\begin{array}{c}\text { SCS } \\
\text { efficiency } \\
{[\%]}\end{array}$ & $\begin{array}{c}\text { ACS } \\
\text { efficiency } \\
{[\%](\mathrm{ES})}\end{array}$ & $\begin{array}{c}\text { ACS } \\
\text { efficiency } \\
{[\%] \text { (MS) }}\end{array}$ \\
\hline$\overline{{ }^{46} \mathrm{Ar}^{17+}}$ & 8400 & 364(10) & $369+122$ & $15(5)$ & $73(1$ & \\
\hline${ }^{46} \mathrm{~K}^{18+}$ & 105000 & ) & 36 & 6( & $98(1$ & $61(9)$ \\
\hline${ }^{37} \mathrm{~K}^{17+}$ & 1220 & $569(10)$ & $369+122$ & $8(4)$ & $79(9)$ & \\
\hline${ }^{34} \mathrm{Ar}^{15+}$ & 845 & $347(10)$ & 125 & $26(3)$ & & 72 \\
\hline${ }^{47} \mathrm{~K}^{17+}$ & 17500 & $2(10)$ & $350+100$ & 21(4) & $65(9)$ & $65(9)$ \\
\hline
\end{tabular}

ejection times. Figure 10 shows the SCS efficiencies of the most abundant charge states of two of these beams: ${ }^{34} \mathrm{Ar}$ and ${ }^{47} \mathrm{~K}$. They were determined by changing the field strength of the magnet of the $Q / A$ separator, and measuring the $\beta$ activity of each charge state at the mass slit. Note that in Fig. 6, the ${ }^{39} \mathrm{~K}$ breeding time was approximately $110 \mathrm{~ms}$. The charge state with the maximum abundance was then 15+. In Fig. 10, as a result of the long breeding time of $350 \mathrm{~ms}$, the peak charge state was shifted to $17+$, an electronic closed-shell (He-like). The SCS efficiencies were calculated from the ratio of the beam activity measured at the mass slit (FC/DC 4) to the activity of the injected beam measured after the BCB (FC/DC 1). The
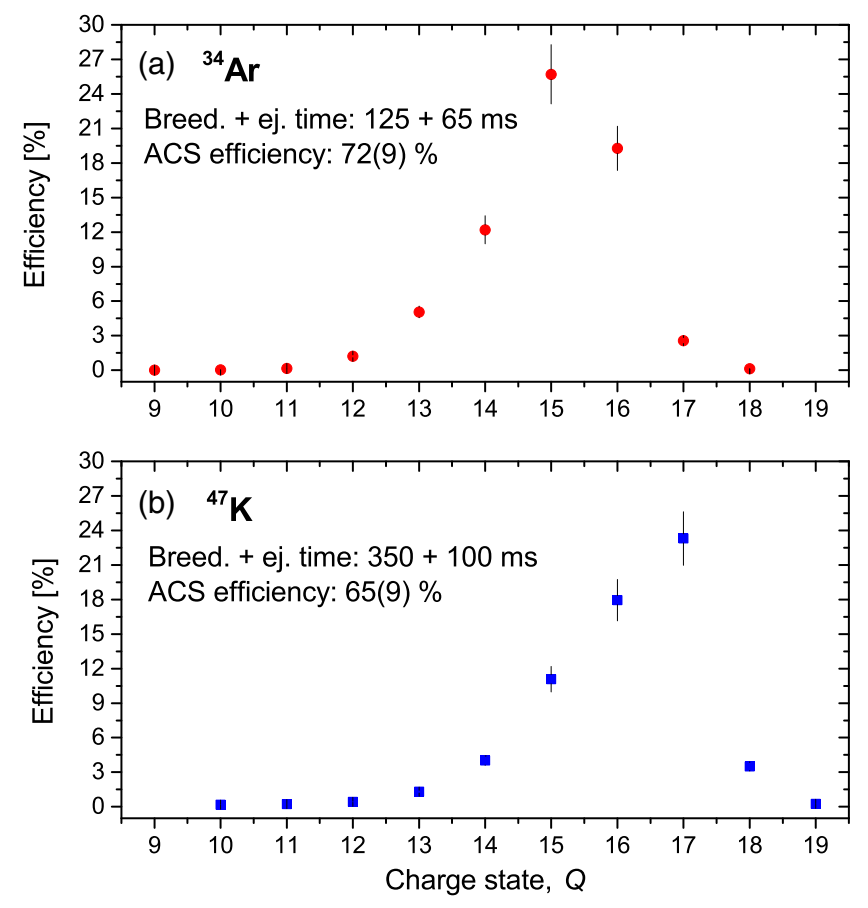

FIG. 10. Efficiency in single charge states (SCS) for (a) ${ }^{34} \mathrm{Ar}$ and (b) ${ }^{47} \mathrm{~K} \cdot{ }^{34} \mathrm{Ar}{ }^{15+}$ and ${ }^{47} \mathrm{~K}^{17+}$ were reaccelerated and delivered to nuclear-physics experiments. Also included in these plots are the all-charge-state (ACS) efficiencies calculated by integrating the SCS efficiencies.
ACS efficiencies were evaluated using two different methods. The ACS efficiencies at the energy slit were calculated from the ratio of the activity measured after the electrostatic sector (FC/DC 3), before charge-state separation, to the activity of the injected beam measured before EBIT injection (FC/DC 1). Note that these ACS efficiencies may include $1+$ ions that were captured in the EBIT, but did not interact with the electron beam during the charge breeding process. The ACS efficiencies measured at the mass slit, after mass separation, were determined by summing the activity of the most abundant charge states after the $Q / A$ scans. They were calculated from the ratio of the activity measured at the energy slit (FC/DC 4) to the activity at the exit of the BCB (FC/DC 1).

The trajectory of the beams ejected from the EBIT can sometimes drift, reducing the transport efficiency through the $Q / A$ separator and, therefore, the rates delivered to the experiments. During beam delivery, the capture, charge breeding, and transport efficiency of the beams are maximized and measured many times. The efficiencies presented in Table III are the average values of several of the best results taken toward the end of the beam times when the transport efficiencies through the many subsystems (e.g., BCB, EBIT, $Q / A$, RFQ, LINAC) were optimized. For ${ }^{34} \mathrm{Ar}$ and ${ }^{46} \mathrm{~K}$, the ACS efficiency measured at the mass slit was obtained by adjusting the integrated efficiency over all charge states for the efficiency of the reaccelerated charge state to match the best-result average. The $Q / A$ scan presented in Fig. 10(a) was obtained after performing this adjustment. All SCS and ACS efficiencies were corrected for losses due to nuclear decays during the breeding period. Decay losses during ion ejection were also considered by taking half the ejection time as an average. The reason for correcting the SCS and ACS efficiency measurements obtained with the rare-isotope beams is to assess these efficiencies independently of the nuclear-decay losses for comparison to CBSIM and efficiency measurements with stable-isotope beams. This is to better assess the atomicphysics processes in the trap (electron-impact ionization, charge-exchange recombination, etc.), ion losses from the trap by evaporation, as well as the injection and ejection efficiencies. For most cases, correcting for the nucleardecay losses increases the ACS and SCS efficiencies by less than 2\%, within the experimental error bars. Only exceptions are ${ }^{37} \mathrm{~K}$ [half-life $=1220 \mathrm{~ms}$ ] and ${ }^{34} \mathrm{Ar}$ [half-life $=845 \mathrm{~ms}$ ] for which the ACS efficiency increases from $65 \%$ to $79 \%$ and the SCS efficiency increases from $23 \%$ to $26 \%$, respectively. When necessary, the efficiencies were also corrected for the dead time of the detection system, of up to $\sim 30 \%$ for high rates. The uncertainty of the efficiency measurements was obtained from the standard deviation of the best results to which a $10 \%$ error was added. This error accounts for possible uncertainties caused by the detectors' different noise levels. The error is more than an order of magnitude larger than the 
statistical error of each measurement. It was estimated from the portion of the low-energy distribution of the $\beta$-decay electrons that may not have been detected by the decay counters because of high noise. The efficiency measurements are therefore mostly dominated by systematic errors such as detector noise and improper beam injection or ejection.

Overall, the measured efficiencies are in satisfactory agreement with one another and consistent with the results obtained with stable ${ }^{39} \mathrm{~K}$. The efficiencies obtained for hydrogen-like ${ }^{46} \mathrm{Ar}^{17+}$ and ${ }^{46} \mathrm{~K}^{18+}$ of $15(5) \%$ and $6(1) \%$, respectively, are significantly different from each other, outside their respective error bars. Based on CBSIM, for the same breeding time and current density, ${ }^{46} \mathrm{~K}^{18+}$ should have an efficiency approximately $80 \%$ that of the ${ }^{46} \mathrm{Ar}^{17+}$. During the runs, the ACS efficiency of both beams was measured to be comparable. Hence, an explanation for the lower ${ }^{46} \mathrm{~K}^{18+}$ efficiency compared to ${ }^{46} \mathrm{Ar}^{17+}$ may be differences in the breeding process. Maximum SCS efficiencies of 26(3)\% and 21(4)\% were obtained with ${ }^{34} \mathrm{Ar}^{15+}$ and ${ }^{47} \mathrm{~K}^{17+}$, respectively. Correcting for the ACS efficiencies of $72 \%$ and $65 \%$, the SCS efficiencies expected if ion losses were not present are $36 \%$ and $32 \%$. Our experimental results for ${ }^{34} \mathrm{Ar}^{15+}$ and ${ }^{47} \mathrm{~K}^{17+}$ are in very close agreement with the loss-corrected measured ${ }^{39} \mathrm{~K}^{15+}$ SCS efficiency of $31 \%$. CBSIM simulations predict SCS efficiencies of $42 \%$ and $47 \%$ for the experimental ratios of ${ }^{34} \mathrm{Ar}^{15+} /{ }^{34} \mathrm{Ar}^{16+} \sim 2.2$ and ${ }^{47} \mathrm{~K}^{17+} /{ }^{47} \mathrm{~K}^{18+} \sim 6.7$, respectively. All experimental efficiencies, even after correcting for ion losses, are significantly lower than those obtained from CBSIM. The broad experimental charge-state distributions are believed to be due to improper injection and trapping (Sec. III A 2). The ACS efficiencies obtained with the rare-isotope beams are generally lower than those of the stable isotopes. The rare-isotope beams are normally of low intensity and some isotopes have long half-lives. As a result, the decay rates measured with the counters can often be low and have long response times. This makes the measured rates less sensitive to changes in the injection and ejection optics, limiting optimization within constrained times.

\section{ELECTRON-BEAM CURRENT DENSITY}

The effective current density of electron beams (the density experienced by the trapped ions) can be estimated by comparing charge-state distributions measured with stable- and rare-isotope beams to CBSIM simulations. This is shown in Table IV, where this density is labeled as "CSD density" In each case, the density was obtained by calculating with CBSIM the product of the current density and breeding time $\left(J_{e} \tau_{B}\right)$ needed to reach a given chargestate ratio, used as an anchor, and dividing by the experimental breeding time. This breeding time was approximated as the sum of the times the ions were confined in the trapping potential closed and half the time during which the trap was opened for ion release. As
TABLE IV. Density (effective) of the electron beam for different electron currents estimated from experimental charge-state distributions (CSD density) using CBSIM with fixed chargestate anchors. These estimates are compared with the density calculated from the measured electron-beam radius (Radius density) [26].

\begin{tabular}{lccccc}
\hline \hline $\begin{array}{l}\text { Element } \\
\text { Isotope }\end{array}$ & $\begin{array}{c}\text { Elec. } \\
\text { current } \\
{[\mathrm{mA}]}\end{array}$ & $\begin{array}{c}\text { Breeding+ } \\
\text { Ejection } \\
\text { time }[\mathrm{ms}]\end{array}$ & $\begin{array}{c}\mathrm{CS} \\
\text { anchor } \\
\text { ratio }\end{array}$ & $\begin{array}{c}\text { CSD } \\
\text { density } \\
{\left[\mathrm{A} / \mathrm{cm}^{2}\right]}\end{array}$ & $\begin{array}{c}\text { Radius } \\
\text { density } \\
{\left[\mathrm{A} / \mathrm{cm}^{2}\right]}\end{array}$ \\
\hline Stable & & & & & \\
${ }^{39} \mathrm{~K}$ & $302(10)$ & $110+23$ & $15 / 16: 1.6$ & 158 & $171(32)$ \\
${ }^{85} \mathrm{Rb}$ & $333(10)$ & $110+20$ & $27 / 28: 2.6$ & 275 & $189(35)$ \\
${ }^{133} \mathrm{Cs}$ & $573(10)$ & $110+23$ & $36 / 36: 1$ & 453 & $325(60)$ \\
$\mathrm{Rare}$ & & & & & \\
${ }^{46} \mathrm{~K}$ & $367(10)$ & $369+122$ & $17 / 18: 3.7$ & 146 & $208(39)$ \\
${ }^{34} \mathrm{Ar}$ & $347(10)$ & $125+65$ & $15 / 16: 2.2$ & 152 & $197(36)$ \\
${ }^{47} \mathrm{~K}$ & $332(10)$ & $350+100$ & $17 / 18: 6.7$ & 117 & $188(35)$ \\
\hline \hline
\end{tabular}

mentioned earlier, a charge-state anchor was obtained from the ratio of the most abundant charge state in a distribution to the least abundant of the two adjacent charge states. For Cs, the two charge states next to $36+$ have approximately equal abundances; the $J_{e} \tau_{B}$ value was chosen to maximize the $36+$ abundance. Note that the CBSIM version used here does not provide data for $\mathrm{Rb}$. The values in Table IV for $\mathrm{Rb}$ result from simulations for $\mathrm{Ge}, \mathrm{Kr}$, and Mo using the $22 / 23,26 / 27$, and $32 / 33$ anchor ratios, respectively, as equal to 2.6, and interpolating to $\mathrm{Rb}$. In [26], the current density of an 800-mA electron beam was derived from an $80 \%$ beam-radius measurement to be $454(83) \mathrm{A} / \mathrm{cm}^{2}$. The column labeled "Radius density" shows the density obtained from the factor of $0.5675\left(\mathrm{~A} / \mathrm{cm}^{2}\right) / \mathrm{mA}$ (as discussed in Sec. II C). As the radius of the electron beam is constant, its current density is proportional to the electron current. During the charge breeding process, partial neutralization of the electron beam by residual gas and the injected charge contribute to lowering the current density experienced by the trapped ions (i.e., the effective current density) by reducing the radial confinement provided by the electron beam. Off-axis ion-beam injection can lead to trapped ion orbits partially outside the electron beam, reducing the effective current density as well. Ions of high temperature can also experience a lower effective current density because high energy ions can leave the volume of the electron beam.

Apart from $\mathrm{Rb}$ and $\mathrm{Cs}$, for which a significant amount of ions might have remained trapped for a few injection/ ejection cycles, all current densities estimated from CBSIM are lower but in satisfactory agreement with the densities inferred from the measured electron-beam radius given the limits of the simulation model. For instance, CBSIM calculates the charge-state evolution of ions fully confined within the electron-beam volume, assumed to have a constant current density in the radial direction, which is 
different from the experimental case. In [29], the density of the ReA EBIT electron beam was simulated to have a nonuniform radial distribution. CBSIM does not also include neutralization with ions from the residual gas and/or injected charge.

Ion-beam injection slightly off-axis and partial neutralization of the electron beam are the expected reasons for the small deviation of the CBSIM-based CSD density from the density inferred from the measured electron-beam radius. Despite that, the two independent methods, the determination of the current density through an electron-beam radius measurement and the determination of the current density by comparing experimental charge-state distributions to simulations, are in satisfactory agreement, indicating that both methods can be used for estimating the electron-beam current density.

\section{STRETCHING OF EJECTED ION PULSES}

The natural width of the ion pulses ejected from the ReA EBIT obtained by lowering the ejection barrier potential with a step voltage function in time is typically in the order of tens of $\mu \mathrm{s}$. Because of the long dead time of detection systems, the instantaneous rate (defined as the ratio of the number of ejected ions to the pulse width) of such short ion pulses accelerated to experiments is often too high for detection of all ions or related events (within each pulse). The pulses must be spread out in time. Stretching the pulses can be accomplished by lowering the ejection barrier potential over a long time period with a time-dependent voltage function resembling a ramp. Theoretical studies were conducted in [35] and test results using this stretching scheme were presented in $[21,30]$. Highly charged ions produced from injected and residual gas, ${ }^{84} \mathrm{Kr}^{26+}$ and ${ }^{40} \mathrm{Ar}^{16+}$, respectively, were used for these tests by detecting ions with an MCP located after the $Q / A$ separator. Pulses were stretched by up to 2 and $9 \mathrm{~ms}$.

The ions in the trap can be assumed to have a MaxwellBoltzmann energy distribution [32]. The maximum of the distribution in the potential well is governed by the ratio of their charge to their temperature. The ions in high charge states, for instance, are deeply bound to the trapping potential. The use of a linear function can release these ions only toward the end of the ejection time, when the ejection barrier potential is near its minimum. For instance, ${ }^{46} \mathrm{Ar}^{17+}$ and ${ }^{46} \mathrm{~K}^{18+}$ ejected with a linear function over $120 \mathrm{~ms}$ were observed to exit the trap within a narrow distribution of approximately 10-20 ms half-way or over the last portion of the ramp. To maximize the ion spread (and make efficient use of the time available for ejection), the barrier potential has to be first lowered rapidly at the beginning of the ejection time to reach the maximum of the energy distribution in the trapping potential [35]. Then, the lowering rate must be reduced for a slow release of ions as needed. This can be realized with exponential or logarithmic functions such as

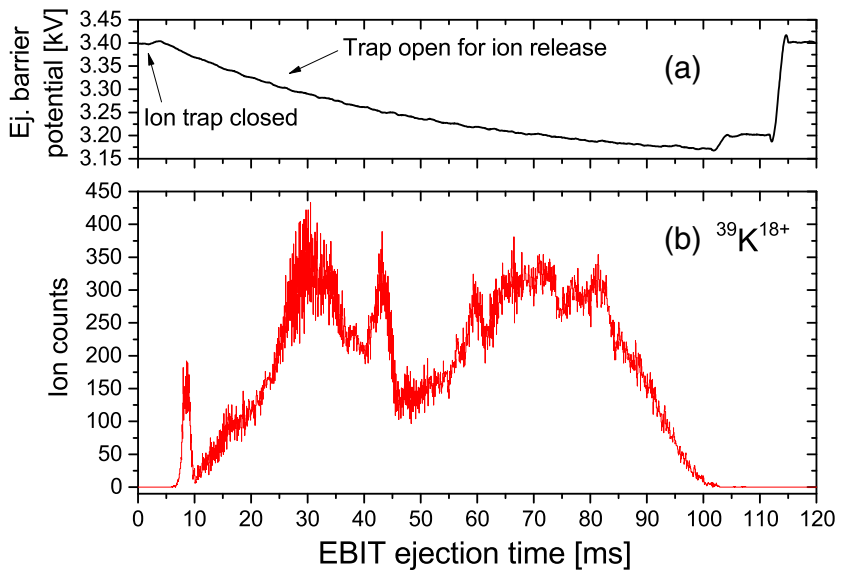

FIG. 11. (a) Exponential voltage ramp function used for stretching ${ }^{39} \mathrm{~K}^{18+}$ ion pulses ejected from the ReA EBIT, (b) stretched ion time distribution measured with an MCP used by an experiment.

$V_{e b}(t)=\left(V_{e b}^{\circ}-V_{e b}^{F}\right) \exp [-\alpha t]+V_{e b}^{F} \quad$ and $\quad V_{e b}(t)=$ $-\gamma \ln \left[\alpha t+\exp \left[-V_{e b}^{\circ} / \gamma\right]\right]$. Here, $V_{e b}(t)$ is the timedependent axial potential applied to the ejection barrier. $V_{e b}^{\circ}$ and $V_{e b}^{F}$ are the ejection barrier potentials at $t=0$ (during charge breeding) and at the end of the ejection time, respectively. $\alpha$ and $\gamma$ are adjustable parameters. Figures 11 and 12 show stretched pulses, using such functions, that were accelerated and delivered to experiments, where the ions were detected with an MCP and a silicon detector. The waveforms displayed in a) were those output by the AFG connected to the control input of the high-voltage amplifier of the ejection barrier electrode. The sharp peak at the very beginning of the ion distributions is due to the sudden change in voltage applied to the ejection barrier potential when the applied voltage becomes controlled by the AFG.

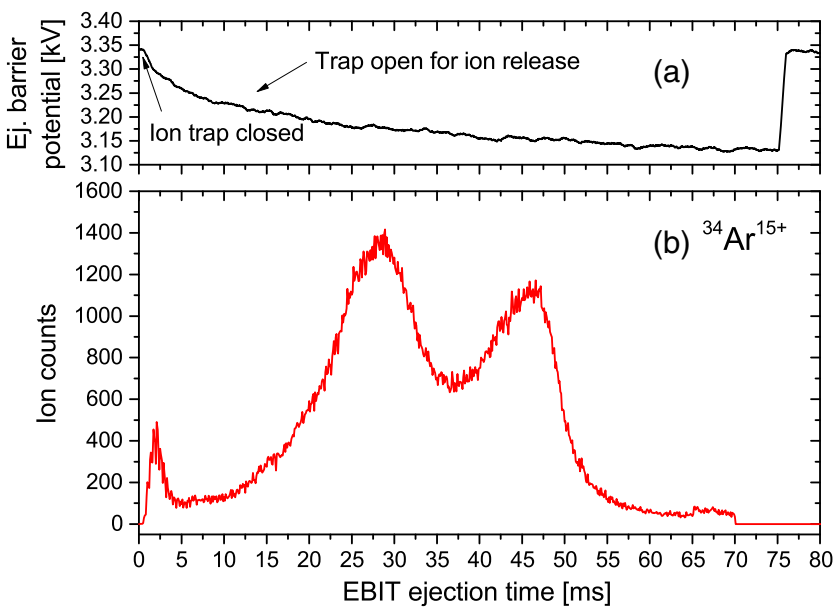

FIG. 12. (a) Logarithmic voltage ramp function used for stretching ${ }^{34} \mathrm{Ar}^{15+}$ ion pulses ejected from the ReA EBIT, (b) stretched ion time distribution measured with a silicon detector used by an experiment. 
The two large maxima are likely the result of a non-uniform central potential having two different potential regions.

Using long ejection ramps does not appear to significantly alter the ejection efficiency. The currents of ${ }^{40} \mathrm{Ar}^{16+}$ and ${ }^{40} \mathrm{Ar}^{17+}$ beams were measured with a Faraday cup after reacceleration before and after changing the ejection mode from a step function to ramp functions of about $120 \mathrm{~ms}$ long. Losses in beam current between $10 \%$ and $20 \%$ were observed. However, these losses can mainly be attributed to the associated decrease in charge breeding time from 489 to $369 \mathrm{~ms}$. Losses due to a change in the ion trajectory of the ejected ions were also observed by changing the ejection ramp function while keeping the ejection time constant. These losses could be recovered by reoptimizing the trajectory of the ejected beam.

Note that using a similar ejection technique as ours, in [36], ${ }^{136} \mathrm{Xe}^{44+}$ pulses were extended up to $50-100 \mathrm{~ms}$. However, unlike in our work, ${ }^{136} \mathrm{Xe}$ is a long-lived isotope. Injecting stable or long-lived isotopes does not impose a strong requirement on the extraction efficiency as these isotopes are abundant. In this publication, the extraction efficiency was not measured and hence was unknown because ${ }^{136} \mathrm{Xe}$ was injected as gas. This is different from our EBIT system for which the extraction (or ejection) efficiency is critical and efficiencies (e.g., charge breeding and ejection) were measured.

\section{CONTAMINATION MEASUREMENTS}

Rare-isotope beams of low intensities (e.g., 1000 particles per second) can easily be obscured by the tail of residual-gas contaminants of several pico-Amperes. Predicting the abundance of contaminants over the $Q / A$ landscape is essential to select a clean region and a charge state of a rare isotope to be accelerated for an experiment. To identify the various stable-isotope contaminants in the ReA EBIT, several $Q / A$ regions were studied over the last years with high sensitivity.

Measuring beam intensities with a Faraday cup or an MCP directly located after the $Q / A$ separator provides information limited to the charge-over-mass ratio of the beam contaminants. This yields insufficient information on the contaminants as elements of many different masses can be charge bred to the same or similar $Q / A$ ratios. The energy of the ions accelerated through the ReA RFQ is a fixed $600 \mathrm{keV} / \mathrm{u} \times A$. Scanning the field strength of the $Q / A$ separator's magnet while measuring the energy of single ions accelerated by the RFQ with an (energydispersive) silicon detector allows the ion charge as well as the atomic mass of the contaminants to be disentangled. A short $Q / A$ region investigated with this method was presented in [22]. Figure 13 presents an $A$-vs- $Q / A$ contamination map of the region from $Q / A=0.375$ to 0.355 , showing the presence of $\mathrm{B}, \mathrm{F}, \mathrm{Ne}, \mathrm{Al}, \mathrm{Si}, \mathrm{Cl}, \mathrm{Ar}$, and $\mathrm{Cu}$ isotopes. Note that in this figure the beam was

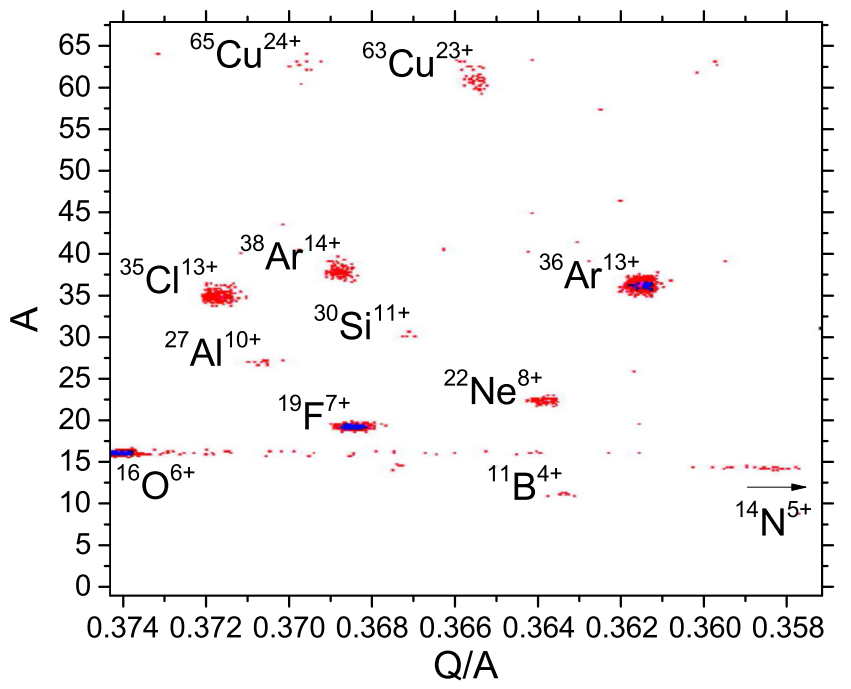

FIG. 13. Map of stable-isotope contaminants obtained by scanning the field strength of the $Q / A$ separator's magnet while reaccelerating beams through the ReA RFQ to $600 \mathrm{keV} / \mathrm{u} \times A$ and measuring the energy of single ions with a silicon detector.

attenuated for the detector to receive less than 1000 ions per second.

A difficulty with the previous method, however, is the beam intensity has to be kept low to limit the dose level received by the silicon detector that induces damage over time. If a $Q / A$ region contains a strong contaminant, the weak contaminants within the same region can be hard to identify with low statistics when the total count rate is restricted to $\sim 1000$ counts per second. Moreover, the RFQ has a finite energy acceptance. Because of the need for attenuation and the RFQ transport efficiency not being constant over a large $Q / A$ region being scanned, the absolute beam intensity determined through this method can have large uncertainties. Similarly, a $Q / A$ scan conducted with an MCP placed after the separator can reach high sensitivity, but the efficiency of the MCP can sometimes be uncertain depending on the beam energy, the type of beam ( $Q$ and $A$ ), and its degree of deterioration after long-time usage. The beam-current measurements with high sensitivity using a Faraday cup is currently being investigated to quantify more accurately the abundance of ejected contaminants. In addition, this method can be useful for detection of beams of rare isotopes of long half-lives difficult to detect with decay counters. Figure 14 shows the same $Q / A$ region presented in Fig. 13 obtained by scanning the field strength of the $Q / A$ separator's magnet and measuring fA-level beam currents downstream using a high-sensitivity, low-noise ammeter. The smallest peak feature in the scan appears to be ${ }^{22} \mathrm{Ne}^{8+}$ of a current of about 2.5 efA, corresponding to approximately 1950 ions per second. ${ }^{22} \mathrm{Ne}^{8+}$ is also observed in the $A$-vs- $Q / A$ contamination map (Fig. 13).

All stable isotopes of $\mathrm{C}, \mathrm{N}, \mathrm{O}$, and Ar from residual gas (air) are the strongest contaminants found in the ReA EBIT, 


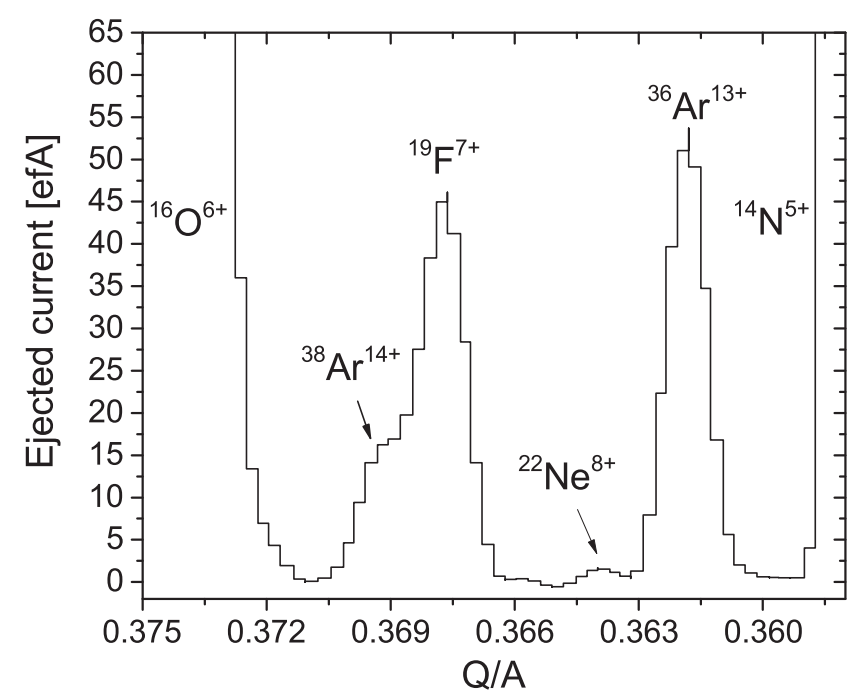

FIG. 14. Stable-isotope contaminants studied by scanning the field strength of the $Q / A$ separator's magnet while measuring ultra-low (efA level) beam currents with a Faraday cup.

ranging in beam intensity from $10^{8}$ ions per second for ${ }^{14} \mathrm{~N}^{6+}$ to $\sim 10^{4}$ ions per second for ${ }^{36} \mathrm{Ar}^{13+}$. F, Na, Si, S, and $\mathrm{Cl}$ are also observed in the range of $10^{4}\left({ }^{19} \mathrm{~F}^{7+}\right)-10^{3}$ $\left({ }^{35} \mathrm{Cl}^{12+}\right)$ ions per second for the most abundant isotopes along with lesser amounts of $\mathrm{B}, \mathrm{Ne}, \mathrm{Al}, \mathrm{K}, \mathrm{Ni}, \mathrm{Cu}, \mathrm{Kr}$, and $\mathrm{Rb}$ isotopes. $\mathrm{K}$ and $\mathrm{Rb}$ originate from the most commonly injected pilot beams.

\section{VII. “CHARGE-SENSITIVE" EJECTION}

In the trap, the ions experience an axial trapping potential proportional to their charge as $Q V_{a t} . V_{a t}$ is the difference in electrostatic potential applied between the center of the trap and the lowest of the two trapping barrier electrodes, which, in our case, is the ejection barrier [32]. For two ion species of different charge states, but identical temperature, the ions of higher charge are expected to be confined to the bottom of the potential well and those of lower charge, being less bound, to live in a region of high potential. When stretching pulses over tens of ms, the ions of low charge state have been observed to be released first. Figure 15 shows the time distribution of residual-gas ${ }^{13} \mathrm{C}^{6+}$ and ${ }^{35} \mathrm{Cl}^{16+}$ directly measured after the $Q / A$ separator with an $\mathrm{MCP}$ and ejected with the same logarithmic voltage ramp function. Both ion species have slightly different $Q / A$ values and are well resolved by the $Q / A$ separator. The two time distributions were measured separately by changing the field strength of the $Q / A$ separator's magnet. All parameters of the trap and ion transport optics were kept constant. In Fig. 15, the background of each beam observed away from the optimal $Q / A$ position was subtracted from the data.

The separation in release time of different ion species is an important observation as this can be employed to reduce the

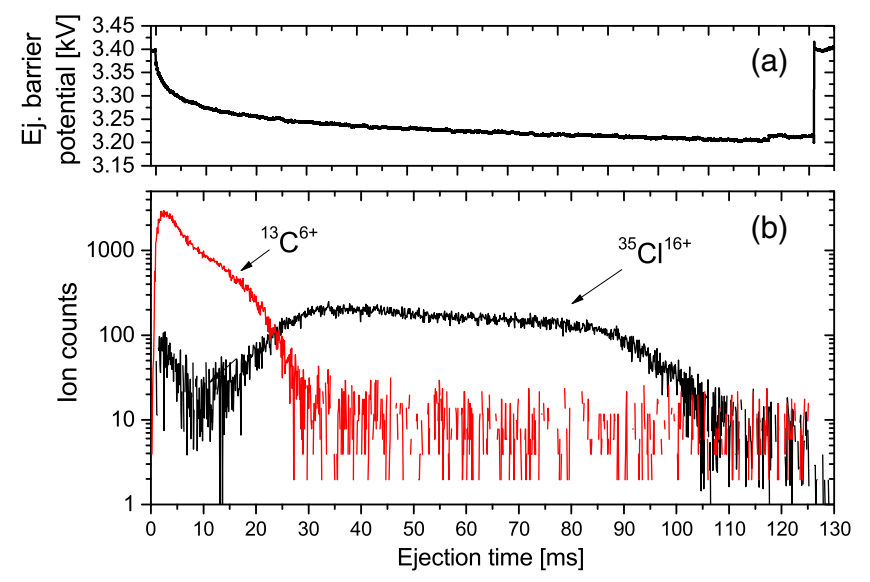

FIG. 15. (a) Logarithmic voltage ramp function for ion ejection, and (b) stretched ion time distribution of ejected ${ }^{13} \mathrm{C}^{6+}$ and ${ }^{35} \mathrm{Cl}^{16+}$ measured after the $Q / A$ separator with an MCP.

amount of contaminants delivered to experiments. Figure 16 presents the time distribution of ${ }^{13} \mathrm{C}^{5+}$ and ${ }^{39} \mathrm{~K}^{15+}$ ions, ejected with an exponential ramp function, reaccelerated, and measured with the MCP of an experiment. Figure 17 shows the EBIT trapping potential during the experiment, indicating the position of the ejection barrier potential at the time the release of ${ }^{13} \mathrm{C}^{5+}$ and ${ }^{39} \mathrm{~K}^{15+}$ peaked. The time distribution containing only ${ }^{13} \mathrm{C}^{5+}$ (EBIT contaminants) was obtained by blocking injection of ${ }^{39} \mathrm{~K}$ from the $\mathrm{BCB}$. This figure also shows a "chopped" ${ }^{39} \mathrm{~K}^{15+}$ time distribution that was purified by delaying the on-time of the RFQ to prevent acceleration of ${ }^{13} \mathrm{C}^{5+}$. This plot shows that approximately $20 \%$ of the ${ }^{39} \mathrm{~K}^{15+}$ intensity was lost to reduce the level of ${ }^{13} \mathrm{C}^{5+}$ contamination by an order of magnitude. Although two ion distributions may not be fully resolved in time and

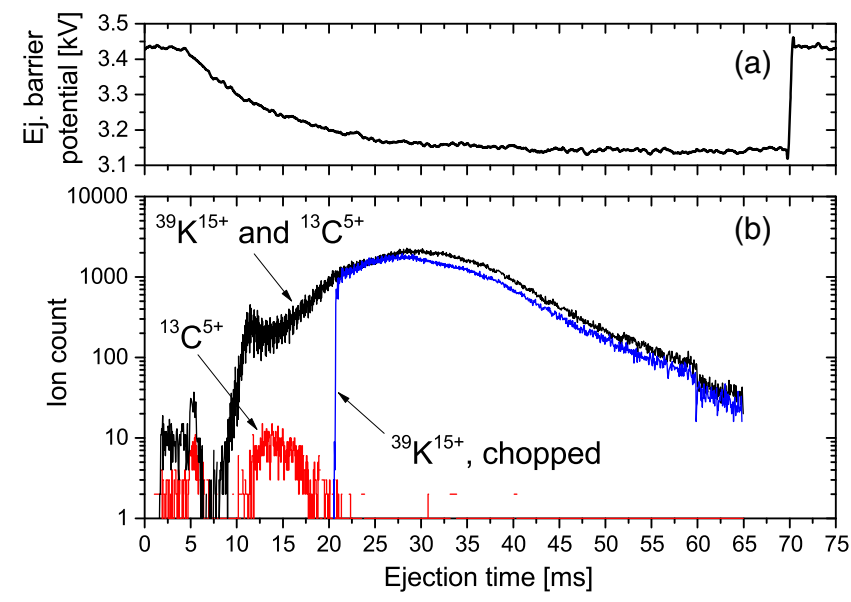

FIG. 16. (a) Exponential voltage ramp function for ion ejection, and (b) ion time distribution of ejected ${ }^{13} \mathrm{C}^{5+}$ and ${ }^{39} \mathrm{~K}^{15+}$ accelerated to several $\mathrm{MeV} / \mathrm{u}$, delivered to a nuclear-physics experiment, and measured with an MCP. The "chopped" ${ }^{39} \mathrm{~K}^{15+}$ time distribution was purified by delaying the on-time of the RFQ to prevent acceleration of ${ }^{13} \mathrm{C}^{5+}$. 


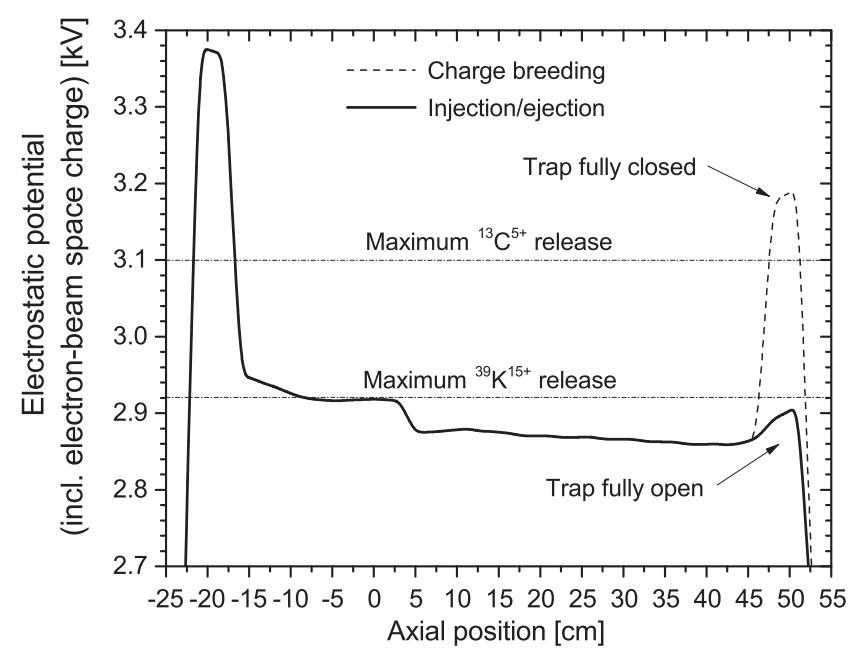

FIG. 17. Trap potential, including the space-charge potential of a 300-mA electron beam, used to obtain the ion distributions shown in Fig. 16. The plot indicates the position of the potentials at the time the release of ${ }^{13} \mathrm{C}^{5+}$ and ${ }^{39} \mathrm{~K}^{15+}$ is maximum.

may overlap at the beginning of the ejection period, cutting out the time distribution of contaminants can be a powerful technique when ion losses are affordable. Depending on the beam energy after acceleration, this novel cleaning technique can outperform the use of a stripper foil, for instance, that can distribute the number of ions in the beam over several charge states and, as a result, inherently induce ion losses.

\section{CONCLUSION AND FUTURE WORK}

The EBIT charge breeder of the ReA post-accelerator has been in operation since September 2015. Efficiencies in single charge states of more than $20 \%$ were measured with ${ }^{39} \mathrm{~K}^{15+},{ }^{85} \mathrm{Rb}^{27+},{ }^{47} \mathrm{~K}^{17+}$, and ${ }^{34} \mathrm{Ar}^{15+}$ with overall efficiencies between $65 \%$ and $89 \%$. The different loss mechanisms that have reduced the overall efficiencies are (1) a decrease in the confinement efficiency of the electron-beam spacecharge (radial) and axial potentials due to partial neutralization; (2) losses caused by ions gaining sufficient energy due to ion-electron collisions and ionization heating to overcome the potential wells (radial and axial); and (3) the injected beam emittance being larger than the acceptance. Those mechanisms are currently being investigated.

Pulses ejected from the EBIT were stretched from a natural $\sim 25 \mu$ s up to $\sim 70 \mathrm{~ms}$ and studies were performed to identify as well as quantify stable-isotope contaminants with high sensitivity. When stretching pulses, ions of low charge state were observed to be released first compared to those of higher charge. A novel beam cleaning method was tested by cutting out the ion time distribution of a contaminant of low charge state, which improved beam purity by an order of magnitude.

Among priorities, future plans include stable operation with a 1-A electron beam, to increase the current density, and spreading out the ejected ion time distributions more uniformly.

\section{ACKNOWLEDGMENTS}

This material is based on work supported by the National Science Foundation under Grant No. PHY-1565546.

[1] A. C. C. Villari, D. Alt, G. Bollen, D. B. Crips, M. Ikegami, S. W. Krause, A. Lapierre, S. M. Lidia, D. J. Morrissey, S. Nash, R. J. Rencsok, R. Ringle, S. Schwarz, R. Shane, C. Sumithrarachchi, S. J. Williams, and Q. Zhao, Commissioning and first accelerated beams in the ReAccelerator (ReA3) of the national superconducting cyclotron facility, MSU, IPAC2016 Proc., http:accelconf.web.cern.ch/ accelconf/ipac2016/index.html.

[2] W. Wittmer, D. Alt, T. M. Baumann, C. Benatti, S. W. Krause, A. Lapierre, D. Leitner, L-Y Lin, F. Montes, S. Nash, R. Rencsok, J. A. Rodriguez, M. Syphers, E. Tanke, and $\mathrm{X}$. Wu, Results from the linac commissioning of the rare isotope reaccelerator-ReA, PAC-2013 Proc., http: accelconf.web.cern.ch/AccelConf/pac2013.

[3] A. Lapierre, S. Schwarz, K. Kittimanapun, J. Fogleman, S. Krause, S. Nash, R. Rencsok, L. Tobos, G. Perdikakis, M. Portillo, J. A. Rodriguez, W. Wittmer, X. Wu, G. Bollen, D. Leitner, and M. Syphers, The ReA electron-beam ion trap charge breeder for reacceleration of rare isotopes, AIP Conf. Proc. 1525, 497 (2013).

[4] D. Leitner, State-of-the-art post-accelerators for radioactive beams, Nucl. Instrum. Methods Phys. Res., Sect. B 317, 235 (2013).

[5] D. Leitner et al., Status of the rare isotope reaccelerator facility ReA, PAC-2013 Proc., http:accelconf.web.cern.ch/ AccelConf/pac2013.

[6] D. J. Morrissey and B. M. Sherrill, In-flight separation of projectile fragments, Lect. Notes Phys. 651, 113 (2004).

[7] P. N. Ostroumov, A. Barcikowski, C. A. Dickerson, A. Perry, A. I. Pikin, S. I. Sharamentov, R. C. Vondrasek, and G. P. Zinkann, Fast and efficient charge breeding of the Californium rare isotope breeder upgrade electron beam ion source, Rev. Sci. Instrum. 86, 083311 (2015).

[8] A. Lapierre, M. Brodeur, T. Brunner, S. Ettenauer, A. T. Gallant, V. Simon, M. Good, M. W. Froese, J. R. Crespo López-Urrutia, P. Delheij, S. Epp, R. Ringle, S. Schwarz, J. Ullrich, and J. Dilling, The TITAN EBIT charge breeder for mass measurements on highly charged short-lived isotopes-First online operation, Nucl. Instrum. Methods Phys. Res., Sect. A 624, 54 (2010).

[9] F. J. C. Wenander, Charge breeding of radioactive ions with EBIS and EBIT, J. Instrum. 5, C10004 (2010).

[10] F. J. C Wenander, Charge Breeding of Radioactive Ions, Contribution to the CAS-CERN Accelerator School: Ion Sources, Senec, Slovakia, 2012, edited by R. Bailey, Report No. CERN-2013-007, arXiv:1404.0945.

[11] F. Ames, M. Marchetto, A. Mjøs, and A.C. Morton, Charge state breeding experiences and plans at TRIUMF, Rev. Sci. Instrum. 87, 02B501 (2016). 
[12] J. Kim, J. E. Han, H. J. Son, K. H. Yi, L. Zhao, and J. S. Kim, An EBIS system for rare isotope science project in Korea, AIP Conf. Proc. 1640, 38 (2015).

[13] D. Rodriguez et al., MATS and LaSpec: High-precision experiments using ion traps and lasers at FAIR, Eur. Phys. J. Spec. Top. 183, 1 (2010).

[14] P. Delahaye, O. Kester, C. Barton, T. Lamy, M. MarieJeanne, and F. Wenander, Evaluation of charge breeding options for EURISOL, Eur. Phys. J. A 46, 421 (2010).

[15] A. Shornikov, E. Beebe, M. Breitenfeldt, J. Pitters, R. Mertzig, A. Pikin, and F. Wenander, Conceptual design report on a charge breeder for HIE-ISOLDE, Report No. CERN-ACC-NOTE-2016-0073, arXiv:1701.00687.

[16] A. Lapierre et al., Commissioning results of the ReA EBIT charge breeder at the NSCL: First reacceleration of stableisotope beams, Nucl. Instrum. Methods Phys. Res., Sect. B 317, 399 (2013).

[17] A. Lapierre, S. Schwarz, T. M. Baumann, K. Cooper, K. Kittimanapun, A. J. Rodriguez, C. Sumithrarachchi, S. J. Williams, W. Wittmer, D. Leitner, and G. Bollen, First charge breeding of a rare-isotope beam with the electronbeam ion trap of the ReA post-accelerator at the National Superconducting Cyclotron Laboratory, Rev. Sci. Instrum. 85, 02 B701 (2014).

[18] A. Lapierre, G. Bollen, J. R. Crespo López-Urrutia, M. Doleans, S. Geyer, O. Kester, K. Kittimanapun, M. Portillo, and S. Schwarz, The MSU EBIT at NSCL, J. Instrum. 5, c07001 (2010).

[19] S. Schwarz, T. M. Baumann, K. Kittimanapun, A. Lapierre, and A. Snyder, A high-current electron gun for the electron beam ion trap at the National Superconducting Cyclotron Laboratory, Rev. Sci. Instrum. 85, $02 B 705$ (2014).

[20] M. Portillo, G. Bollen, S. Chouhan, O. Kester, G. Machicoane, J. Ottarson, S. Schwarz, and A. Zeller, in Proceedings of the 23rd Particle Accelerator Conference, Vancouver, Canada, 2009 (IEEE, Piscataway, NJ, 2009).

[21] T. M. Baumann, A. Lapierre, S. Schwarz, K. Kittimanapun, and G. Bollen, Energy spread and time structure of ion beams extracted from the ReA-EBIT rare isotope charge breeder, AIP Conf. Proc. 1640, 80 (2015).

[22] S. J. Williams, T. Baumann, K. Cooper, A. Lapierre, D. Leitner, D. J. Morrissey, G. Perdikakis, J. A. Rodriguez, S. Schwarz, A. Spyrou, M. Steiner, C. Sumithrarachchi, and W. Wittmer, in IBIC2013 Proc., edited by I. Martin and G. Rehm (Diamond Light Source, Oxford, 2013), p. 19, ISBN: 978-3-95450-127-4.

[23] P. Mandal, G. Sikler, and M. Mukherjee, Simulation study and analysis of a compact einzel lens-deflector for low energy ion beam, J. Instrum. 6, P02004 (2011).
[24] http://simion.com/.

[25] The emittance is defined as the phase-space area of the beam divided by $\pi$. The root-mean-square (rms) emittance in one $x$ dimension is defined as $\epsilon_{x, \mathrm{rms}}=$ $\sqrt{\left\langle x^{2}\right\rangle\left\langle x^{\prime 2}\right\rangle+\left\langle x x^{\prime}\right\rangle^{2}}$, where $\left\langle x^{2}\right\rangle,\left\langle x^{\prime 2}\right\rangle$, and $\left\langle x x^{\prime}\right\rangle^{2}$ are the moments of the particle distribution in the $x-x^{\prime}$ phase space as in M. Reiser, Theory and Design of Charged Particle Beams (John Wiley and Sons, Inc., New York, 1994).

[26] T. M. Baumann, A. Lapierre, K. Kittimanapun, S. Schwarz, D. Leitner, and G. Bollen, Determination of the ReA Electron Beam Ion Trap electron beam radius and current density with an X-ray pinhole camera , Rev. Sci. Instrum. 85, 073302 (2014).

[27] R. E. Marrs, Self-cooling of highly charged ions during extraction from electron beam ion sources and traps, Nucl. Instrum. Methods Phys. Res., Sect. B 149, 182 (1999).

[28] C. Dickerson, B. Mustapha, A. Pikin, S. Kondrashev, P. Ostroumov, A. Levand, and R. Fischer, Simulation and design of an electron beam ion source charge breeder for the californium rare isotope breeder upgrade, Phys. Rev. ST Accel. Beams 16, 024201 (2013).

[29] K. Kittimanapun, T. M. Baumann, A. Lapierre, S. Schwarz, and G. Bollen, Transverse acceptance calculation for continuous ion beam injection into the electron beam ion trap charge breeder of the ReA post-accelerator, Nucl. Instrum. Methods Phys. Res., Sect. A 800, 57 (2015).

[30] S. Schwarz and A. Lapierre, Recent charge-breeding developments with EBIS/T devices (invited), Rev. Sci. Instrum. 87, 02A910 (2016).

[31] B. R. Barquest, G. Bollen, P. F. Mantica, K. Minamisono, R. J. Ringle, S. Schwarz, and C. S. Sumithrarachchi, RFQ beam cooler and buncher for collinear laser spectroscopy of rare isotopes, Nucl. Instrum. Methods Phys. Res., Sect. A 866, 18 (2017).

[32] F. J. Currell and G. Fussmann, Physics of electron beam ion traps and sources, IEEE Trans. Plasma Sci. 33, 1763 (2005).

[33] G. Herrmann, Optical theory of thermal velocity effects in cylindrical electron beams, J. Appl. Phys. 29, 127 (1958).

[34] R. Becker, O. Kester, and Th. Stoehlker, Simulation of charge breeding for trapped ions, J. Phys. Conf. Ser. 58, 443 (2007).

[35] A. Lapierre, Time-dependent potential functions to stretch the time distributions of ion pulses ejected from EBIST, Can. J. Phys. 95, 361 (2017).

[36] E. Beebe, L. Liljeby, A. Engström, and M. Björkhage, The Stockholm electron beam ion source, Phys. Scr. 47, 470 (1993). 